\title{
A Fixed Point Theorem and Equivariant Points for Set-valued Mappings
}

\author{
By
}

Yoshimi SHITANDA*

\begin{abstract}
We give a proof of a coincidence theorem for a Vietoris mapping and a compact mapping and prove the Lefschetz fixed point theorem for the class of admissible mappings which contains upper semi-continuous acyclic mappings. When a source space is a paracompact Hausdorff space with a free involution and a target space is a closed topological manifold with an involution, the existence of equivariant points is proved for the class of admissible mappings under some conditions. When a source space is a Poincaré space with a finite covering dimension, the covering dimension of the set of equivariant points is determined.
\end{abstract}

\section{§1. Introduction}

S. Eilenberg and D. Montgomery [1] gave the Lefschetz fixed point formula for acyclic mappings which is a generalization of the classical Lefschetz fixed point theorem. L. Górniewicz [7] studied set-valued mappings, a coincidence theorem for ANR spaces and the Lefschetz fixed point theorem for admissible mappings. M. Nakaoka studied the Lefschetz fixed point theorem by the cohomological method in [12] and equivariant point theorems [14, 16] and the Borsuk-Ulam theorem between manifolds in $[13,16]$. In this paper, the author shall give a proof of a coincidence theorem for a Vietoris mapping and a compact mapping (cf. Definition 3.2) and prove the Lefschetz fixed point theorem for the class of admissible mappings (cf. Definition 3.5). We shall generalize many results of M. Nakaoka [16] to set-valued mappings between a

\footnotetext{
Communicated by S. Mukai. Received March 7, 2008. Revised June 23, 2008, August 26, 2008, October 14, 2008, January 14, 2009.

2000 Mathematics Subject Classification(s): 55M20, 55N05.

*Meiji University, Izumi Campus, Eifuku 1-9-1, Suginami-ku, Tokyo 168-8555, Japan.
} 
paracompact Hausdorff space with a free involution and a closed manifold with an involution.

In general, when a non-empty closed set $\varphi(x)$ in a topological space $Y$ is assigned for every point $x$ in a topological space $X$, we say that the correspondence is a set-valued mapping and write $\varphi: X \rightarrow Y$ by the Greek alphabet. For single-valued mapping, we write $f: X \rightarrow Y$ etc. by the Roman alphabet. A set-valued mapping is studied particularly in Chapter 2 in [7]. In this paper we assume that any set-valued mapping is upper semi-continuous.

In the section 2, we shall discuss various cohomology theories and state some results for our applications. We shall mainly use the Alexander-Spanier cohomology theory $\bar{H}^{*}(-; \mathbf{F})$ with coefficient in a field $\mathbf{F}$ instead of the singular cohomology theory $H^{*}(-; \mathbf{F})$.

In the section 3, we shall prove a fixed point theorem for set-valued mappings. For the purpose, we shall prove the following theorem (cf. Theorem 3.9) which is our main theorem whose proof is different from L. Górniewicz [6, 7].

Main Theorem 1. Let $X$ be an ANR space and $Y$ a paracompact Hausdorff space. Let $p: Y \rightarrow X$ be a Vietoris mapping and $q: Y \rightarrow X$ a compact mapping. Then $\left(p^{*}\right)^{-1} q^{*}$ is a Leray endomorphism. If the Lefschetz number $L\left(\left(p^{*}\right)^{-1} q^{*}\right)$ is not zero, there exists a coincidence point $z \in Y$, that is, $p(z)=q(z)$.

For an admissible mapping $\varphi: X \rightarrow Y$, we define $\varphi^{*}: \bar{H}^{*}(Y ; \mathbf{F}) \rightarrow$ $\bar{H}^{*}(X ; \mathbf{F})$ by the set $\left\{\left(p^{*}\right)^{-1} q^{*}\right\}$ where $(p, q)$ is a selected pair of $\varphi$. From the above theorem, we obtain the following Lefschetz fixed point theorem (cf. Theorem 3.10).

Main Theorem 2. Let $X$ be an ANR space and $\varphi: X \rightarrow X$ a compact admissible mapping. If $L\left(\varphi^{*}\right)$ contains a non-trivial element, there exists a fixed point $x_{0} \in X$, that is, $x_{0} \in \varphi\left(x_{0}\right)$.

In the section 4, we shall prove the Steenrod isomorphism (cf. Theorem 4.1) for compact ANR spaces of finite type and the Alexander-Spanier cohomology theory. We also define cohomology operations $P_{i}(-)$ and $P(-,-)$ for general spaces and deduce the naturalities with respect to mappings. We discuss the Gysin-Smith sequence for the Alexander-Spanier cohomology theory and its properties. We shall prove a result on Poincaré spaces for our purposes (cf. Lemma 4.4). Poincaré space is a connected metric space satisfying the Poincaré duality.

We define the equivariant fundamental cohomology class $\hat{U}_{M} \in \bar{H}^{m}\left(S^{\infty} \times_{\pi}\right.$ $\left.\left(M^{2}, M^{2}-\Delta M\right) ; \mathbf{F}_{2}\right)$ for a closed manifold $M$ with an involution which is a 
connected compact manifold without boundary. For a paracompact Hausdorff space $N$ with a free involution $T$ and a continuous mapping $f: N \rightarrow M$, we obtain a result on the set $A(f)=\left\{x \in N \mid f(T(x))=T^{\prime}(f(x))\right\}$ of equivariant points (cf. Theorem 4.5) and evaluate the dimension of $A(f)$ for a Poincaré space $N$ with a finite covering dimension (cf. Theorem 4.6).

M. Nakaoka proved some equivariant point theorems (cf. Theorem 5.3 in [14], Theorem 7.1 etc. in [16]) between the same dimensional closed manifolds. In the section 5, we shall generalize his results for set-valued mappings between a Poincaré space with a finite covering dimension and a closed manifold.

The generalized Lefschetz number $\mathcal{L}\left(\varphi:\left[N_{\pi, \alpha}\right]\right)$ is defined for an admissible mapping $\varphi: N \rightarrow M$ (cf. Definition 5.2). In general $\mathcal{L}\left(\varphi:\left[N_{\pi, \alpha}\right]\right)$ is a set. Our main theorem is stated as follows (cf. Theorem 5.5).

Main Theorem 3. Let $N$ be a paracompact Hausdorff space with a free involution $T$ and $M$ an $m$-dimensional closed topological manifold with an involution $T^{\prime}$ satisfying the condition (5.1). For an admissible mapping $\varphi$ : $N \rightarrow M$, if $\mathcal{L}\left(\varphi:\left[N_{\pi, \alpha}\right]\right)$ contains a non-trivial element, then there exists a point $x_{0} \in N$ such that $T^{\prime} \varphi\left(x_{0}\right) \cap \varphi\left(T\left(x_{0}\right)\right) \neq \emptyset$. Moreover if $N$ is an $n$ dimensional Poincaré space and the covering dimension of $N$ is finite, it holds $\operatorname{dim} A(\varphi) \geqq n-m$ where $A(\varphi)=\left\{x \in N \mid T^{\prime}(\varphi(x)) \cap \varphi(T(x)) \neq \emptyset\right\}$.

When a source space is the sphere and a target space is the Euclidean space, the Borsuk-Ulam theorem is proved in $\S 37, \S 43$ of [7] and Theorem 2.6 in [4] for admissible mappings. In the section 6 , we shall generalize their Borsuk-Ulam theorem to the case of general spaces (cf. Theorem 6.3). For a paracompact Hausdorff space $X$ with a free involution $T$, let $X_{\pi}$ be the orbit space of $X$ by the group $\pi$ generated by $T$. The first Stiefel-Whitney class $c=c(X, T)$ of $X$ is defined by $c=f^{*}(\omega)$ where $f: X_{\pi} \rightarrow R P^{\infty}$ is the classifying mapping of the projection $p: X \rightarrow X_{\pi}$ and $\omega$ is the generator of $H^{1}\left(R P^{\infty} ; \mathbf{F}_{2}\right)$.

Main Theorem 4 . Let $N$ be a paracompact Hausdorff space with a free involution $T$ and $M$ an $m$-dimensional closed topological manifold. Assume that the first Stiefel-Whitney class $c(N, T)$ satisfies $c(N, T)^{m} \neq 0$. If a setvalued mapping $\varphi: N \rightarrow M$ is admissible and $\varphi^{*}$ contains the trivial element, then there exists a point $x_{0} \in N$ such that $\varphi\left(x_{0}\right) \cap \varphi\left(T\left(x_{0}\right)\right) \neq \emptyset$. Moreover if $N$ is an $n$-dimensional Poincaré space and the covering dimension of $N$ is finite, it holds $\operatorname{dim} A(\varphi) \geqq n-m$ where $A(\varphi)=\{x \in N \mid \varphi(x) \cap \varphi(T(x)) \neq \emptyset\}$.

From our theorem, we can obtain the detailed results of the Borsuk-Ulam theorem for admissible mappings in the case that a source space or a target 
space has the homology groups of the sphere (cf. Corollarys 6.4, 6.5). Moreover we shall determine the index of $A(\varphi)$ of an admissible mapping $\varphi: N \rightarrow$ $M$ in the case that $N$ satisfies $c(N, T)^{n} \neq 0$ (cf. Corollary 6.6). These are generalizations of Theorem 43.11 in L. Górniewicz [7], Theorem 3.4 in K. Gȩba and L. Górniewicz [4].

\section{§2. Various Cohomology Theories}

To begin with, we give some remarks about several cohomology theories. The Alexander-Spanier cohomology theory $\bar{H}^{*}(-; G)$ is isomorphic to the singular cohomology theory $H^{*}(-; G)$ (cf. Theorem 6.9.1 in [17]), that is,

$$
\mu: \bar{H}^{*}(X ; G) \cong H^{*}(X ; G)
$$

if the singular cohomology theory satisfies the continuity:

$$
\lim _{\overrightarrow{\{U\}}} H^{*}(U ; G)=H^{*}(x ; G)
$$

where $\{U\}$ is a system of neighborhoods of $x$. The remarkable feature of the Alexander-Spanier cohomology theory is that it satisfies the continuity property (cf. Theorem 6.6.2 in [17]).

For a paracompact Hausdorff space $X$, it also holds the isomorphism between the Čech cohomology theory $\check{H}^{*}(-; G)$ with coefficient in a constant sheaf and the Alexander-Spanier cohomology theory $\bar{H}^{*}(-; G)$ (cf. Theorem 6.8.8 in [17]), that is,

$$
\check{H}^{*}(X ; G) \cong \bar{H}^{*}(X ; G) .
$$

If a normal space $X$ satisfies the property of neighborhood retract for any normal space $Z$ which contains $X$ as a closed subset, it is called an ANR space. An ANR metric space is an $r$-image of some open set of a normed space (cf. Proposition 1.8 in [7]). For an ANR metric space $X$, these three cohomology groups are isomorphic to each other by Theorem 6.1 .10 of [17]. In this paper, we assume that ANR space is a metric space. Hereafter we assume that any (co)homology theory is a (co)homology theory with coefficient in a field $\mathbf{F}$.

For a covering $\mathcal{U}$ of $X$, the simplicial complex $K(\mathcal{U})$ is defined in $\S 1$ of Chapter 3 in [17] and is called the nerve of $\mathcal{U}$. The simplicial complex $X(\mathcal{U})$ is defined in $\S 5$ of Chapter 6 in [17] and is called the Vietoris simplicial complex of $\mathcal{U}$. They are chain equivalent each other (cf. Exercises D of Chapter 6 in 
[17]). Clearly by the definition of the Alexander-Spanier cohomology theory, we have the isomorphism:

$$
\lim _{\{\mathcal{U}\}} H^{*}\left(C^{*}(X(\mathcal{U}) ; \mathbf{F})\right) \cong \bar{H}^{*}(X ; \mathbf{F}) .
$$

We have the cross products $\bar{\tau}: \bar{H}^{*}(X, A ; \mathbf{F}) \otimes \bar{H}^{*}(Y, B ; \mathbf{F}) \rightarrow \bar{H}^{*}((X, A) \times$ $(Y, B) ; \mathbf{F})$ and $\tau: H^{*}(X, A ; \mathbf{F}) \otimes H^{*}(Y, B ; \mathbf{F}) \rightarrow H^{*}((X, A) \times(Y, B) ; \mathbf{F})$ and the natural transformation $\mu: \bar{H}(-; \mathbf{F}) \rightarrow H^{*}(-; \mathbf{F})$. They satisfy the following commutativity:

$$
\begin{array}{ccc}
\bar{H}^{*}(X, A ; \mathbf{F}) \otimes \bar{H}^{*}(Y, B ; \mathbf{F}) & \overline{\bar{\tau}} \\
& \downarrow^{\mu \otimes \mu} & \bar{H}^{*}((X, A ; \mathbf{F}) \times(Y, B) ; \mathbf{F}) \\
H^{*}(X, A ; \mathbf{F}) \otimes H^{*}(Y, B ; \mathbf{F}) \stackrel{\tau}{\longrightarrow} & H^{*}((X, A) \times(Y, B) ; \mathbf{F}) .
\end{array}
$$

For the detail see $\S 5, \S 9$ and Exercise E of Chapter 6 in [17]. If $X$ and $Y$ are ANR spaces and $H_{*}(X, A ; \mathbf{F})$ or $H_{*}(Y, B ; \mathbf{F})$ is finite type, we can easily obtain the Künneth theorem for the Alexander-Spanier cohomology theory. Under the same condition, we can find a cofinal covering system $\{\mathcal{U} \times \mathcal{V}\}$ of $X \times Y$ such that $\bar{H}^{*}(X \times Y ; \mathbf{F}) \cong \lim _{\rightarrow} \bar{H}^{*}(X(\mathcal{U}) \times Y(\mathcal{V}) ; \mathbf{F})$ where $\mathcal{U}$ and $\mathcal{V}$ are open coverings of $X$ and $Y$ respectively. This is easily proved by the above consideration.

The Čech homology theory $\check{H}_{*}(-; \mathbf{F})$ (cf. [2]) and the Alexander-Spanier homology theory $\bar{H}_{*}(X ; \mathbf{F})$ are defined by

$$
\varliminf_{\{\mathcal{U}\}} H_{*}\left(C_{*}(K(\mathcal{U}) ; \mathbf{F})\right), \quad \lim _{\{\mathcal{U}\}} H_{*}\left(C_{*}(X(\mathcal{U}) ; \mathbf{F})\right)
$$

respectively. They are isomorphic to each other (cf. Exercise D in Chapter 6 in [17]). The following theorem is well-known (cf. Theorem 1 in [10]).

Theorem 2.1. Let $X$ be a ANR space. Then it holds the isomorphism:

$$
\check{H}_{*}(X ; \mathbf{F}) \cong H_{*}(X ; \mathbf{F}) .
$$

Since the Alexander-Spanier homology theory coincides with the singular homology theory for ANR space, it holds the following isomorphism:

$$
\bar{H}^{*}(X ; \mathbf{F})=\operatorname{Hom}\left(\bar{H}_{*}(X ; \mathbf{F}), \mathbf{F}\right)
$$

by the universal coefficient theorem of the singular homology theory.

We can obtain the universal coefficient theorem for the Alexander-Spanier (co)homology theory. 
Lemma 2.2. Let $X$ be a compact Hausdorff space. Then it holds the isomorphism:

$$
\bar{H}_{*}(X ; \mathbf{F}) \cong \operatorname{Hom}\left(\bar{H}^{*}(X ; \mathbf{F}), \mathbf{F}\right) .
$$

Proof. Since there exists a cofinal family $\{\mathcal{U}\}$ of finitely many open sets for a compact set $X$, we have the universal coefficient theorem:

$$
\begin{aligned}
\bar{H}_{*}(X ; \mathbf{F}) \cong \lim _{\overleftarrow{\mathcal{U}}} H_{*}\left(C_{*}(K(\mathcal{U}) ; \mathbf{F})\right) \\
\cong \lim _{\overleftarrow{\mathcal{U}}} \operatorname{Hom}\left(H^{*}\left(C^{*}(K(\mathcal{U}) ; \mathbf{F}), \mathbf{F}\right)\right) \\
\cong \operatorname{Hom}\left(\lim _{\overrightarrow{\mathcal{U}}} H^{*}\left(C^{*}(K(\mathcal{U}) ; \mathbf{F}), \mathbf{F}\right)\right) \\
\cong \operatorname{Hom}\left(\bar{H}^{*}(X ; \mathbf{F}), \mathbf{F}\right) .
\end{aligned}
$$

Here the second isomorphism is obtained by the universal coefficient theorem for chain complexes of finite type. The third isomorphism is given by the isomorphism: $\lim _{\overleftarrow{\alpha}} \operatorname{Hom}\left(F_{\alpha}, \mathbf{F}\right) \cong \operatorname{Hom}\left(\lim _{\vec{\alpha}} F_{\alpha}, \mathbf{F}\right)$ for $\mathbf{F}$-vector spaces $F_{\alpha}$.

For open coverings $\mathcal{U}_{\beta}, \mathcal{U}_{\alpha}$ of $X$, the notation $\mathcal{U}_{\beta}<\mathcal{U}_{\alpha}$ means that $\mathcal{U}_{\beta}$ is finer than $\mathcal{U}_{\alpha}$.

Lemma 2.3. Let $X$ be a compact $A N R$ space of finite type $\bar{H}_{*}(X ; \mathbf{F})$. Then there exist finitely many open coverings $\mathcal{U}_{\alpha}$ and $\mathcal{U}_{\beta}$ of $X$ such that $\mathcal{U}_{\beta}<$ $\mathcal{U}_{\alpha}$ and it holds:

$$
\bar{H}_{*}(X ; \mathbf{F}) \cong \text { image of }\left\{H_{*}\left(K\left(\mathcal{U}_{\beta}\right) ; \mathbf{F}\right) \rightarrow H_{*}\left(K\left(\mathcal{U}_{\alpha}\right) ; \mathbf{F}\right)\right\} .
$$

Proof. Set $V=\bar{H}_{*}(X ; \mathbf{F})$ and $V_{\alpha}=H_{*}\left(K\left(\mathcal{U}_{\alpha}\right) ; \mathbf{F}\right)$. Define $V_{\alpha}^{\prime}$ and $K_{\alpha}^{\prime}$ by the image of $V \rightarrow V_{\alpha}$ and the kernel of $V \rightarrow V_{\alpha}$ respectively. Set $L_{\alpha}=V_{\alpha} / V_{\alpha}^{\prime}$. There exist exact sequences:

$$
0 \rightarrow\left\{K_{\alpha}^{\prime}\right\} \rightarrow\{V\} \rightarrow\left\{V_{\alpha}^{\prime}\right\} \rightarrow 0, \quad 0 \rightarrow\left\{V_{\alpha}^{\prime}\right\} \rightarrow\left\{V_{\alpha}\right\} \rightarrow\left\{L_{\alpha}\right\} \rightarrow 0 .
$$

And we obtain the following exact sequences:

$$
0 \rightarrow \lim _{\leftarrow} K_{\alpha}^{\prime} \rightarrow V \rightarrow \lim _{\leftarrow} V_{\alpha}^{\prime} \rightarrow \cdots, \quad 0 \rightarrow \lim _{\leftarrow} V_{\alpha}^{\prime} \rightarrow \lim _{\leftarrow} V_{\alpha} \rightarrow \lim _{\leftarrow} L_{\alpha} \rightarrow \cdots
$$

Since it holds $V=\lim _{\leftarrow} V_{\alpha}$ by the definition of the Alexander-Spanier homology theory, we have $V=\lim _{\leftarrow} V_{\alpha}^{\prime}$ and $\lim _{\leftarrow} K_{\alpha}^{\prime}=0$. From this, there exists $\mathcal{U}_{\beta}$ for each $\mathcal{U}_{\alpha}$ such that $K_{\beta}^{\prime}=0$ and $\mathcal{U}_{\beta}<\mathcal{U}_{\alpha}$. Therefore we may 
consider the cofinal system $\left\{V \rightarrow V_{\alpha}\right\}$ of mono-morphisms. Here note that $\bar{H}_{*}(X ; \mathbf{F})$ is finite type. By making use of Lebesgue's covering theorem, we may assume that $\left\{V_{\alpha}\right\}$ is a countable linear ordered system. From the exact sequence $0 \rightarrow\{V\} \rightarrow\left\{V_{\alpha}\right\} \rightarrow\left\{M_{\alpha}\right\} \rightarrow 0$ and $V=\lim _{\leftarrow} V_{\alpha}$, we have $\lim _{\leftarrow} M_{\alpha}=0$. Therefore we easily see that there exists a refinement $\mathcal{U}_{\beta}$ for each $\mathcal{U}_{\alpha}$ such that $V$ is isomorphic to the image of $i_{\alpha \beta}: V_{\beta} \rightarrow V_{\alpha}$.

\section{$\S 3 . \quad$ A Coincidence Theorem}

We shall give a proof of a coincidence theorem in this section. Our proof is different from the one of L. Górniewicz [6, 7] and depends on the line of M. Nakaoka [12]. In this paper, we work in the category of paracompact Hausdorff spaces and continuous mappings.

Let $U$ be an open set in the $n$-dimensional Euclidean space $\mathbf{R}^{n}$ and $K$ a compact subset of $U$. We have the following diagram:

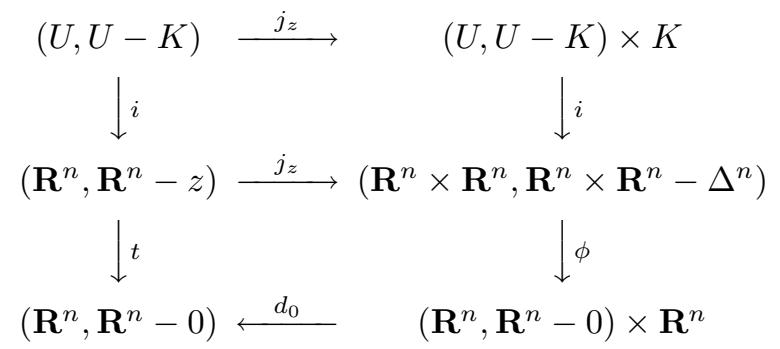

where $j_{z}(x)=(x, z), z \in K, t(x)=x-z, \phi(x, y)=(x-y, y), d_{0}(x, y)=x$ and $\Delta^{n}$ the diagonal set of $\mathbf{R}^{n} \times \mathbf{R}^{n}$.

Let $w_{x}$ be the generator of $\bar{H}_{n}\left(\mathbf{R}^{n}, \mathbf{R}^{n}-x ; \mathbf{F}\right)$ corresponding to $1 \in \mathbf{F}$. Then there exists the generator $w_{K}^{U}$ of $\bar{H}_{n}(U, U-K ; \mathbf{F})$ such that $\left(j_{K}^{U}\right)_{*}\left(w_{K}^{U}\right)=$ $w_{x}$ for any $x \in K$ and the inclusion $j_{K}^{U}:(U, U-K) \rightarrow\left(\mathbf{R}^{n}, \mathbf{R}^{n}-x\right)$. Note that $\bar{H}_{n}(U, U-K ; \mathbf{F}) \cong H_{n}(U, U-K ; \mathbf{F})$ for a compact set $K$. We shall use only the case of finite complex $K$ for our application. The class $\gamma_{x}$ is defined by the generator of $\bar{H}^{n}\left(\mathbf{R}^{n}, \mathbf{R}^{n}-x ; \mathbf{F}\right)$ which is the dual element of $w_{x}$. Naturally, we denote $w_{0}$ and $\gamma_{0}$ for $x=0$.

Definition 3.1. Define the class $\gamma_{K}^{U} \in \bar{H}^{n}((U, U-K) \times K ; \mathbf{F})$ by $\gamma_{K}^{U}=$ $i^{*} \phi^{*} d_{0}^{*}\left(\gamma_{0}\right)$ where $d_{0} \phi i:(U, U-K) \times K \rightarrow\left(\mathbf{R}^{n}, \mathbf{R}^{n}-0\right)$.

The following definition is essentially important for our purpose. 
Definition 3.2. $\quad$ Let $X$ and $Y$ be paracompact Hausdorff spaces. A mapping $f: X \rightarrow Y$ is called a Vietoris mapping, if it satisfies the following conditions:

1. $f$ is proper and onto continuous mapping.

2. $f^{-1}(y)$ is an acyclic space for any $y \in Y$, that is, $\bar{H}^{*}\left(f^{-1}(y) ; \mathbf{F}\right)=0$ for positive dimension.

When $f$ is closed and onto continuous mapping and satisfies the condition (2), we call it a weak Vietoris mapping.

If $f^{-1}(K)$ is compact set for any compact subset $K \subset Y, f$ is called a proper mapping. Note that a proper mapping is closed. Our definition is broader than L. Górniewicz's, because he works in the category of metric spaces. The following Vietoris theorem holds only for the Alexander-Spanier cohomology theory (cf. Theorem 6.9.15 in [17]).

Theorem 3.1. Let $f: X \rightarrow Y$ be a weak Vietoris mapping between paracompact Hausdorff spaces $X$ and $Y$. Then,

$$
f^{*}: \bar{H}^{m}(Y ; \mathbf{F}) \rightarrow \bar{H}^{m}(X ; \mathbf{F})
$$

is an isomorphism for all $m \geqq 0$.

In this paper, we redefine expediently the Alexander-Spanier homology theory by the following equation:

$$
\bar{H}_{*}(X ; \mathbf{F})=\operatorname{Hom}\left(\bar{H}^{*}(X ; \mathbf{F}), \mathbf{F}\right)
$$

instead of the equation (2.5). From the properties of $\bar{H}^{*}(-; \mathbf{F})$, the new homology theory $\bar{H}_{*}(-; \mathbf{F})$ satisfies the axioms of generalized homology theory. These two definitions for the Alexander-Spanier homology theory coincide each other for ANR spaces of finite type or compact Hausdorff spaces. Let $K$ be a compact set $\left\{x_{m}\right\}_{m \geqq 1} \cup\{z\}$ in $\mathbf{R}^{n}$ where the sequence $\left\{x_{m}\right\}_{m \geqq 1}$ convergences to $z$. $\mathbf{R}^{n}-K$ gives an example that these two homology theories do not coincide. We shall use the new definition (3.3) for the consistency.

We must remark a fundamental fact:

$$
f_{*}: \bar{H}_{m}(X ; \mathbf{F}) \rightarrow \bar{H}_{m}(Y ; \mathbf{F})
$$

is an isomorphism for all $m \geqq 0$ for a Vietoris mapping $f$. A mapping $f: X \rightarrow$ $Y$ is called a compact mapping, if $f(X)$ is contained in a compact set of $Y$, or equivalently its closure $\overline{f(Y)}$ is compact. 
We can take a finite complex $L$ for a compact space $K$ in $U \subset \mathbf{R}^{n}$ such that $K \subset L \subset U$. Because we subdivide $\mathbf{R}^{n}$ into small boxes whose faces are parallel to axes and construct the complex $L$ by collecting small boxes which intersect with $K$. For a finite complex $K$, we have the isomorphism between the Čech homology theory $\check{H}_{*}(K ; \mathbf{F})$ and the singular homology theory $H_{*}(K ; \mathbf{F})$. We use this case for our application in this section.

Definition 3.3. $\quad$ Let $U$ be an open set of the $n$-dimensional Euclidean space $\mathbf{R}^{n}$ and $Y$ a paracompact Hausdorff space. For a weak Vietoris mapping $p: Y \rightarrow U$ and a compact mapping $q: Y \rightarrow U$, the coincidence index $I(p, q)$ of $p$ and $q$ is defined by

$$
I(p, q) w_{0}=\bar{q}_{*}(\bar{p})_{*}^{-1}\left(w_{K}^{U}\right)
$$

where $K$ is a compact set satisfying $q(Y) \subset K \subset U \cdot \bar{p}:\left(Y, Y-p^{-1}(K)\right) \rightarrow$ $(U, U-K)$ and $\bar{q}:\left(Y, Y-p^{-1}(K)\right) \rightarrow\left(\mathbf{R}^{n}, \mathbf{R}^{n}-0\right)$ are defined by $\bar{p}(y)=p(y)$ and $\bar{q}(y)=p(y)-q(y)$ respectively.

If we use the cohomology theory instead of the homology theory in Definition 3.3, we shall be able to prove the corresponding result in the following argument. We use the homology theory because of the comparison with [12] and [6]. From this definition, we easily obtain the next formula.

Lemma 3.2. It holds the formula:

$$
d_{*}\left(1 \times q_{*}\left(p_{*}\right)^{-1}\right) \Delta_{*}\left(w_{K}^{U}\right)=I(p, q) w_{0}
$$

where $\Delta(x)=(x, x), f(y)=(p(y), y), d(x, y)=x-y$.

Proof. We easily obtain the result from the following commutative diagram:

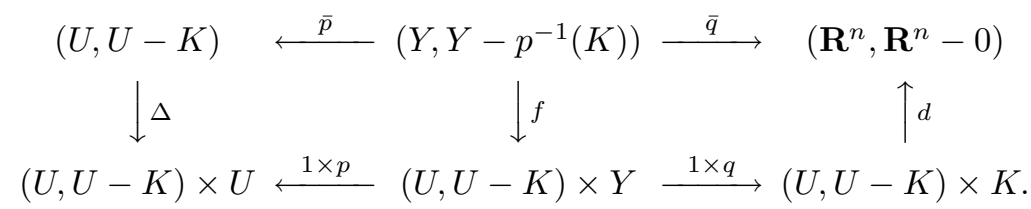

\section{Lemma 3.3.}

$$
I(p, q)=<\Delta^{*}\left(1 \times\left(p^{*}\right)^{-1} q^{*}\right) \gamma_{K}^{U}, w_{K}^{U}>
$$


Proof. We obtain the result from the following calculation:

$$
\begin{aligned}
I(p, q) & =<\gamma_{0}, I(p, q) w_{0}> \\
& =<\gamma_{0}, d_{*}\left(1 \times q_{*}\left(p_{*}\right)^{-1}\right) \Delta_{*}\left(w_{K}^{U}\right)> \\
& =<\Delta^{*}\left(1 \times\left(p^{*}\right)^{-1} q^{*}\right) d^{*}\left(\gamma_{0}\right), w_{K}^{U}> \\
& =<\Delta^{*}\left(1 \times\left(p^{*}\right)^{-1} q^{*}\right) \gamma_{K}^{U}, w_{K}^{U}>
\end{aligned}
$$

Theorem 3.4. $\quad$ Let $U$ be an open set of the $n$-dimensional Euclidean space $\mathbf{R}^{n}$ and $Y$ a paracompact Hausdorff space. For $p: Y \rightarrow U$ a weak Vietoris mapping and $q: Y \rightarrow U$ a compact mapping, if the index $I(p, q)$ is not zero, there exists a coincidence point $z \in Y$, that is, $p(z)=q(z)$.

Proof. Let $K$ be a finite complex of $\mathbf{R}^{n}$ such that $q(Y) \subset K \subset U$. Set

$$
\chi_{p, q}=\left\{x \in U \mid x \in q p^{-1}(x)\right\}, \quad \chi_{p, q}^{\prime}=\{y \in Y \mid p(y)=q(y)\} .
$$

Since $p$ is closed, $\chi_{p, q}$ is a closed set by $p\left(\chi_{p, q}^{\prime}\right)=\chi_{p, q}$. Since $\chi_{p, q}^{\prime}$ is a closed set and $q$ is a compact mapping, $\chi_{p, q}=q\left(\chi_{p, q}^{\prime}\right)$ is a compact set contained in $K$. In the following diagram,

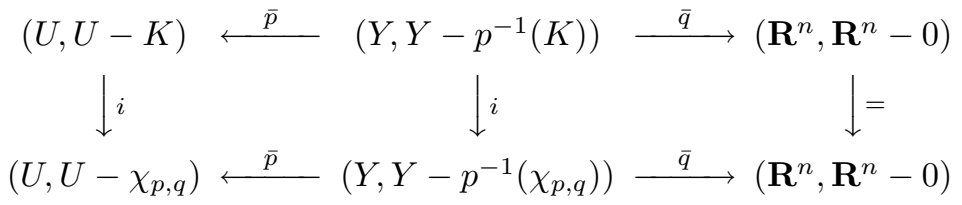

if $\chi_{p, q}$ is an empty set, we obtain $\bar{H}_{n}\left(U, U-\chi_{p, q}\right)=0$ and $I(p, q)=0$ which is the contradiction. Therefore we have the result.

Let $\mathbf{V}$ be a vector space and $f: \mathbf{V} \rightarrow \mathbf{V}$ a linear mapping. Let $f^{k}$ be the $k$ time iterated composition of $f$. Set $N(f)=\cup_{k \geqq 0} \operatorname{ker} f^{k} \subset \mathbf{V}$ and $\tilde{\mathbf{V}}=\mathbf{V} / N(f)$. Then $f$ induces the linear mapping $\tilde{f}: \tilde{\mathbf{V}} \rightarrow \tilde{\mathbf{V}}$ which is a monomorphism. When $\operatorname{dim} \tilde{\mathbf{V}}<\infty$, we define $\operatorname{Tr}(f)$ by $\operatorname{Tr}(\tilde{f})$. In the case of $\operatorname{dim} \mathbf{V}<\infty$, it coincides with the classical trace $\operatorname{Tr}(f)$.

Definition 3.4. Let $\left\{\mathbf{V}_{k}\right\}_{k}$ be a graded vector space and $f=\left\{f_{k}\right.$ : $\left.\mathbf{V}_{k} \rightarrow \mathbf{V}_{k}\right\}_{k}$ a graded linear mapping. Define the generalized Lefschetz number for the case of $\sum_{k \geqq 0} \operatorname{dim} \tilde{\mathbf{V}}_{k}<\infty$ :

$$
L(f)=\sum_{k \geqq 0}(-1)^{k} \operatorname{Tr}\left(f_{k}\right) .
$$


In this case, $f=\left\{f_{k}\right\}_{k}$ is called a Leray endomorphism.

The following elementary result is needed to generalize to a class of general spaces (cf. $\S 11$ in $[7])$.

Lemma 3.5. In the following commutative diagram of graded vector spaces:

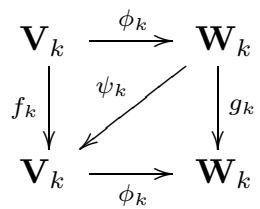

If one of $f=\left\{f_{k}\right\}_{k}$ and $g=\left\{g_{k}\right\}_{k}$ is a Leray endomorphism, the other is also a Leray endomorphism, and $L(f)=L(g)$ holds.

The following theorem is a new proof of a coincidence theorem which is based on M. Nakaoka [12].

Theorem 3.6. $\quad$ Let $U$ be an open set in the $n$-dimensional Euclidean space $\mathbf{R}^{n}$ and $Y$ a paracompact Hausdorff space. Let $p: Y \rightarrow U$ be a weak Vietoris mapping and $q: Y \rightarrow U$ a compact mapping. Then $\left(p^{*}\right)^{-1} q^{*}$ : $H^{*}(U ; \mathbf{F}) \rightarrow H^{*}(U ; \mathbf{F})$ is a Leray endomorphism and we have the following formula:

$$
L\left(\left(p^{*}\right)^{-1} q^{*}\right)=I(p, q) .
$$

Especially, if the Lefschetz number $L\left(\left(p^{*}\right)^{-1} q^{*}\right)$ is not zero, there exists a coincidence point $z \in Y$ such that $p(z)=q(z)$.

Proof. To begin with, we remark that there exists a finite complex $K$ in $U$ such that $q(Y) \subset K \subset U$. Consider the following diagram:

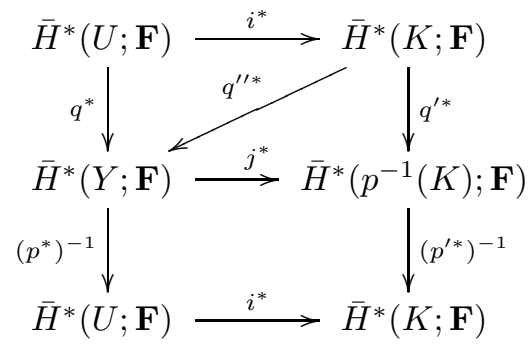

where $p^{\prime}$ and $q^{\prime}$ are the restricted mappings of $p$ and $q$ to the subspace $p^{-1}(K)$ respectively. $q^{\prime \prime}: Y \rightarrow K$ is defined by $q^{\prime}=q^{\prime \prime} j$ and $q=i q^{\prime \prime}$ where $i: K \rightarrow$ 
$U$ and $j: p^{-1}(K) \rightarrow Y$ are the inclusions. Since $\left(p^{* *}\right)^{-1} q^{\prime *}: \bar{H}^{*}(K ; \mathbf{F}) \rightarrow$ $\bar{H}^{*}(K ; \mathbf{F})$ is a Leray endomorphism, $\left(p^{*}\right)^{-1} q^{*}: \bar{H}^{*}(U ; \mathbf{F}) \rightarrow \bar{H}^{*}(U ; \mathbf{F})$ is also a Leray endomorphism by Lemma 3.5. Then, we have

$$
L\left(\left(p^{\prime *}\right)^{-1} q^{\prime *}\right)=L\left(\left(p^{*}\right)^{-1} q^{*}\right) .
$$

Consider the following diagram:

$$
\begin{aligned}
& \bar{H}^{*}(K ; \mathbf{F}) \quad=\quad \bar{H}^{*}(K ; \mathbf{F}) \\
& \downarrow\left(p^{*}\right)^{-1} q^{\prime \prime *} \quad \downarrow\left(p^{* *}\right)^{-1} q^{\prime *} \\
& \bar{H}^{*}(U ; \mathbf{F}) \stackrel{i^{*}}{\longrightarrow} \bar{H}^{*}(K ; \mathbf{F}) \\
& \downarrow(-) \cap w_{K}^{U} \quad \uparrow(-1)^{q} \gamma_{K}^{U} /(-) \\
& H_{*}(U, U-K ; \mathbf{F}) \stackrel{=}{\longrightarrow} H_{*}(U, U-K ; \mathbf{F})
\end{aligned}
$$

Clearly the upper square is commutative for the Alexander-Spanier cohomology theory. The commutativity of the lower square for the singular (co)homology theory is proved by Lemma 3 in [12], that is,

$$
i^{*}(x)=(-1)^{q} \gamma_{K}^{U} /\left(x \cap w_{K}^{U}\right)
$$

for $x \in H^{q}(U ; \mathbf{F})$. Note that we use the sign $(-1)^{q}$ instead of $(-1)^{n q}$ in [12] under some changes of sign (cf. Theorem 12.1 in [11]). Now we shall show the commutativity of the lower square for the Alexander-Spanier cohomology theory. The morphism $i^{*}: \bar{H}^{*}(U ; \mathbf{F}) \rightarrow \bar{H}^{*}(K ; \mathbf{F})$ of the Alexander-Spanier cohomology theory coincides with the one of the singular cohomology theory for a finite complex $K$. So we can determine $i^{*}: \bar{H}^{*}(U ; \mathbf{F}) \rightarrow \bar{H}^{*}(K ; \mathbf{F})$. Note that the Alexander-Spanier cohomology groups $\bar{H}^{*}(U ; \mathbf{F}), \bar{H}^{*}(U, U-$ $K ; \mathbf{F}), \quad \bar{H}^{*}((U, U-K) \times K ; \mathbf{F})$ and $\bar{H}^{*}(K ; \mathbf{F})$ are coincide with ones of the singular cohomology theory.

In the following discussion, we must carefully use the Alexander-Spanier cohomology theory and the singular cohomology theory. Let $\left\{\alpha_{\lambda}\right\},\left\{\beta_{\mu}\right\},\left\{\gamma_{\nu}\right\}$ be basis of $\bar{H}^{*}(U ; \mathbf{F}), \bar{H}^{*}(U, U-K ; \mathbf{F}), \bar{H}^{*}(K ; \mathbf{F})$ respectively. We represent $\gamma_{K}^{U} \in \bar{H}^{*}((U, U-K) \times K ; \mathbf{F})$ as follows:

$$
\gamma_{K}^{U}=\sum_{\mu, \nu} c_{\mu \nu} \beta_{\mu} \times \gamma_{\nu}
$$

Since $p^{*}$ is isomorphic, we set

$$
\left(p^{*}\right)^{-1} q^{\prime \prime *}\left(\gamma_{\xi}\right)=\sum_{\lambda} m_{\lambda \xi} \alpha_{\lambda} .
$$


We calculate the Lefschetz number $L\left(\left(p^{\prime *}\right)^{-1} q^{\prime *}\right)$ :

$$
\begin{aligned}
(-1)^{q}\left(p^{\prime *}\right)^{-1} q^{\prime *}\left(\gamma_{\xi}\right) & =(-1)^{q} i^{*}\left(p^{*}\right)^{-1} q^{\prime \prime *}\left(\gamma_{\xi}\right) \\
& =\gamma_{K}^{U} /\left(\left(p^{*}\right)^{-1} q^{\prime \prime *}\left(\gamma_{\xi}\right) \cap w_{K}^{U}\right) \\
& =\sum_{\mu, \nu} c_{\mu \nu}\left(\beta_{\mu} \times \gamma_{\nu}\right) /\left(\left(p^{*}\right)^{-1} q^{\prime \prime *}\left(\gamma_{\xi}\right) \cap w_{K}^{U}\right) \\
& =\sum_{\mu, \nu} c_{\mu \nu}<\beta_{\mu},\left(p^{*}\right)^{-1} q^{\prime \prime *}\left(\gamma_{\xi}\right) \cap w_{K}^{U}>\gamma_{\nu} \\
& =\sum_{\mu, \nu} c_{\mu \nu}<\beta_{\mu},\left(\sum_{\lambda} m_{\lambda \xi} \alpha_{\lambda}\right) \cap w_{K}^{U}>\gamma_{\nu} \\
& =\sum_{\lambda, \mu, \nu} c_{\mu \nu} m_{\lambda \xi}<\beta_{\mu} \cup \alpha_{\lambda}, w_{K}^{U}>\gamma_{\nu} .
\end{aligned}
$$

Therefore we obtain a formula:

$$
L\left(\left(p^{\prime *}\right)^{-1} q^{\prime *}\right)=\sum_{\lambda, \mu, \xi} c_{\mu \xi} m_{\lambda \xi}<\beta_{\mu} \cup \alpha_{\lambda}, w_{K}^{U}>.
$$

Next we calculate the coincidence index $I(p, q)$ :

$$
\begin{aligned}
I(p, q) & =<\Delta^{*}\left(1 \times\left(p^{*}\right)^{-1} q^{\prime \prime *}\right)\left(\gamma_{K}^{U}\right), w_{K}^{U}> \\
& =\sum_{\mu, \nu} c_{\mu \nu}<\Delta^{*}\left(\beta_{\mu} \times\left(p^{*}\right)^{-1} q^{\prime \prime *}\left(\gamma_{\nu}\right)\right), w_{K}^{U}> \\
& =\sum_{\mu, \nu} c_{\mu \nu}<\Delta^{*}\left(\beta_{\mu} \times\left(\sum_{\lambda} m_{\lambda \nu} \alpha_{\lambda}\right)\right), w_{K}^{U}> \\
& =\sum_{\lambda, \mu, \nu} c_{\mu \nu} m_{\lambda \nu}<\beta_{\mu} \cup \alpha_{\lambda}, w_{K}^{U}>.
\end{aligned}
$$

From these formulas, we have $L\left(\left(p^{\prime *}\right)^{-1} q^{\prime *}\right)=I(p, q)$. Since $L\left(\left(p^{\prime *}\right)^{-1} q^{\prime *}\right)$ is equal to $L\left(\left(p^{*}\right)^{-1} q^{*}\right)$, we obtain the result $L\left(\left(p^{*}\right)^{-1} q^{*}\right)=I(p, q)$. Therefore we obtain the second statement by the above result and Theorem 3.4.

We can generalize the above result to the case of ANR spaces through the line of L. Górniewicz [6, 7]. The following Schauder approximation theorem is useful to generalize for general spaces (cf. Theorem 12.9 in [7], Theorem 2.3 of $\S 6$ in $[8])$.

Theorem 3.7. Let $U$ be an open set of a normed space $\mathbf{E}$ and $f$ : $X \rightarrow U$ a continuous compact mapping. Then, for any $\epsilon>0$, there exists a continuous compact mapping $f_{\epsilon}: X \rightarrow U$ satisfying the following condition: 
1. $f_{\epsilon}(X) \subset \mathbf{E}^{n(\epsilon)}$ for a finite dimensional subspace $\mathbf{E}^{n(\epsilon)}$ of $\mathbf{E}$

2. $\left\|f_{\epsilon}(x)-f(x)\right\|<\epsilon$ for any $x \in X$

3. $f_{\epsilon}(x), f(x): X \rightarrow U$ are homotopic, noted by $f_{\epsilon} \simeq f$.

Theorem 3.8. Let $U$ be an open set in a normed space $\mathbf{E}$ and $Y$ a paracompact Hausdorff space. Let $p: Y \rightarrow U$ be a weak Vietoris mapping and $q: Y \rightarrow U$ a compact mapping. Then $\left(p^{*}\right)^{-1} q^{*}$ is a Leray endomorphism. We assume that the graph of $q p^{-1}$ is closed. If the Lefschetz number $L\left(\left(p^{*}\right)^{-1} q^{*}\right)$ is not zero, there exists a coincidence point $z \in Y$, that is, $p(z)=q(z)$.

Proof. Let $q_{n}: Y \rightarrow U$ be a Schauder approximation of $q$ for $\epsilon=\frac{1}{n}$, that is, $q_{n}(Y) \subset \mathbf{E}^{\epsilon_{n}} \cap U=U_{n}$. Then $q_{n}$ is a compact mapping. $p_{n}: p^{-1}\left(U_{n}\right) \rightarrow U_{n}$ is a weak Vietoris mapping where $p_{n}$ is the restriction of $p$ to $p^{-1}\left(U_{n}\right)$. Here $j_{n}: p^{-1}\left(U_{n}\right) \rightarrow Y$ and $i_{n}: U_{n} \rightarrow U$ satisfy $p j_{n}=i_{n} p_{n}$. Let $q_{n}^{\prime \prime}: Y \rightarrow U_{n}$ be defined by $i_{n} q_{n}^{\prime \prime}=q_{n}$ and $q_{n}^{\prime}: p^{-1}\left(U_{n}\right) \rightarrow U_{n}$ be the restriction of $q_{n}^{\prime \prime}$ to $p^{-1}\left(U_{n}\right)$ i.e. $q_{n}^{\prime \prime} j_{n}=q_{n}^{\prime}$.

Since $\left(p_{n}^{*}\right)^{-1} q_{n}^{\prime *}$ is a Leray endomorphism by Theorem $3.6,\left(p^{*}\right)^{-1} q_{n}^{*}$ is also a Leray endomorphism from the following diagram:

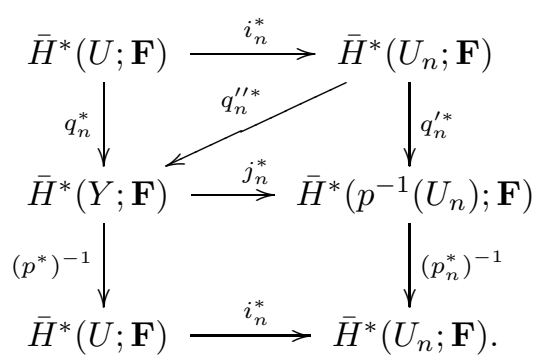

Therefore $\left(p^{*}\right)^{-1} q^{*}$ is also a Leray endomorphism by $q_{n} \simeq q$. And it holds

$$
L\left(\left(p^{*}\right)^{-1} q^{*}\right)=L\left(\left(p^{*}\right)^{-1} q_{n}^{*}\right)=L\left(\left(p_{n}^{*}\right)^{-1}\left(q_{n}^{\prime}\right)^{*}\right) .
$$

By $L\left(\left(p_{n}^{*}\right)^{-1}\left(q_{n}^{\prime}\right)^{*}\right) \neq 0, p_{n}$ and $q_{n}^{\prime}$ have a coincidence point $p_{n}\left(z_{n}\right)=$ $q_{n}^{\prime}\left(z_{n}\right)$ for $z_{n} \in p^{-1}\left(U_{n}\right) \subset Y$ by Theorem 3.6. Since $q$ is a compact map, we can set $x_{0}=\lim _{n \rightarrow \infty} q\left(z_{n}\right)$. By $\left\|q\left(z_{n}\right)-q_{n}\left(z_{n}\right)\right\|<\frac{1}{n}$, we have $x_{0}=$ $\lim _{n \rightarrow \infty} q_{n}\left(z_{n}\right)=\lim _{n \rightarrow \infty} q_{n}^{\prime}\left(z_{n}\right)$ and $x_{0}=\lim _{n \rightarrow \infty} p_{n}\left(z_{n}\right)$. Since it holds $\left(p\left(z_{n}\right), q\left(z_{n}\right)\right) \in \Gamma_{q p^{-1}}$ and $\Gamma_{q p^{-1}}$ is closed, we have $\left(x_{0}, x_{0}\right) \in \Gamma_{q p^{-1}}$. Therefore we see that $p$ and $q$ have a coincidence point $x_{0}$, i.e. $p\left(y_{0}\right)=q\left(y_{0}\right)=x_{0}$ for $y_{0} \in Y$. 
If $p$ is proper in the above theorem, we need not the assumption that the graph of $q p^{-1}$ is closed. An ANR space is a deformation retract of an open set of normed space by Proposition 1.8 in [7], that is, $i: X \rightarrow U, r: U \rightarrow X$ such that $r i=i d_{X}$.

Theorem 3.9. $\quad$ Let $X$ be an $A N R$ space and $Y$ a paracompact Hausdorff space. Let $p: Y \rightarrow X$ be a Vietoris mapping and $q: Y \rightarrow X$ a compact mapping. Then $\left(p^{*}\right)^{-1} q^{*}$ is a Leray endomorphism. If the Lefschetz number $L\left(\left(p^{*}\right)^{-1} q^{*}\right)$ is not zero, there exists a coincidence point $z \in Y$, that is, $p(z)=$ $q(z)$.

Proof. We construct the following diagram where the right square is a pull-back:

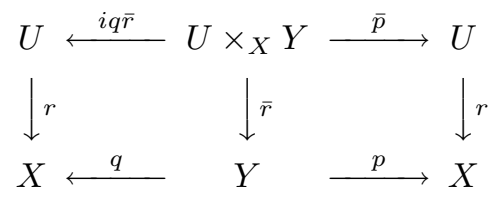

Since $U$ is a paracompact Hausdorff space and $p$ and also $\bar{p}$ are proper, $U \times_{X} Y$ is also a paracompact Hausdorff space. This is proved by Theorem 4 in [9]. Since $p$ is a Vietoris mapping, $\bar{p}$ is also a Vietoris mapping. $\bar{q}=i q \bar{r}$ is a compact mapping. We see that $\left(\bar{p}^{*}\right)^{-1}(\bar{q})^{*}$ is a Leray endomorphism by Theorem 3.8. Therefore we see that $\left(p^{*}\right)^{-1} q^{*}$ is a Leray endomorphism by the above diagram. By $L\left(\left(p^{*}\right)^{-1} q^{*}\right)=L\left(\left(\bar{p}^{*}\right)^{-1}(\bar{q})^{*}\right) \neq 0, \bar{p}$ and $\bar{q}$ have a coincidence point from the Theorem 3.8, that is, $\bar{p}\left(u_{0}, y_{0}\right)=\bar{q}\left(u_{0}, y_{0}\right)$. From this, we easily see that $p$ and $q$ have a coincidence point $y_{0} \in Y$, i.e. $p\left(y_{0}\right)=$ $q\left(y_{0}\right)$.

A set-valued mapping $\varphi: X \rightarrow Y$ is called upper semi-continuous, if for every $x \in X$ and any neighborhood $V$ of $\varphi(x)$, there exists a neighborhood $U$ of $x \in X$ such that $\varphi(U) \subset V$. If $\varphi$ is upper semi-continuous, the graph $\Gamma_{\varphi}=\{(x, y) \in X \times Y \mid y \in \varphi(x)\}$ is a closed set in $X \times Y$. But the converse is not true. If $Y$ is a compact set, the upper semi-continuity of $\varphi$ is equivalent to that the graph $\Gamma_{\varphi}$ is closed.

Definition 3.5. A set-valued mapping $\varphi: X \rightarrow Y$ is called admissible, if it is upper semi-continuous and there exists a paracompact Hausdorff space $\Gamma$ satisfying the following conditions:

1. there exist a Vietoris mapping $p: \Gamma \rightarrow X$ and a continuous mapping $q: \Gamma \rightarrow Y$. 
2. $\varphi(x) \supset q\left(p^{-1}(x)\right)$ for each $x \in X$.

$\varphi: X \rightarrow Y$ is called weak admissible, if it satisfies the condition (2) and $p$ is a weak Vietoris mapping. A pair $(p, q)$ of mappings $p, q$ is called a selected pair of $\varphi$.

An upper semi-continuous set-valued mapping is called acyclic, if $\varphi(x)$ is a closed acyclic space in $Y$ for $x \in X$, that is, $\bar{H}^{*}(\varphi(x) ; \mathbf{F})=0$ for positive dimension. Let $\Gamma_{\varphi}$ be the graph of $\varphi \cdot \varphi(x)$ is considered as $q_{\varphi}\left(p_{\varphi}^{-1}(x)\right)$ where $p_{\varphi}: \Gamma_{\varphi} \rightarrow X$ and $q_{\varphi}: \Gamma_{\varphi} \rightarrow Y$ are defined by $p_{\varphi}(x, y)=x$ and $q_{\varphi}(x, y)=y$ respectively. If $\varphi: X \rightarrow Y$ is compact acyclic mapping, $p_{\varphi}: \Gamma_{\varphi} \rightarrow X$ is a Vietoris mapping. An acyclic mapping $\varphi: X \rightarrow Y$ is admissible. An admissible mapping is not necessarily acyclic mapping. In this case we define uniquely $\varphi^{*}: \bar{H}^{*}(Y ; \mathbf{F}) \rightarrow \bar{H}^{*}(X ; \mathbf{F})$ by $\left(p_{\varphi}^{*}\right)^{-1} q_{\varphi}^{*}$.

Generally $\varphi^{*}: \bar{H}^{*}(Y ; \mathbf{F}) \rightarrow \bar{H}^{*}(X ; \mathbf{F})$ is defined by the set $\left\{\left(p^{*}\right)^{-1} q^{*}\right\}$ where $(p, q)$ is a selected pair of a weak admissible mapping $\varphi: X \rightarrow Y . \varphi_{*}$ is similarly defined. A mapping $\varphi: S^{n} \rightarrow S^{n}$ defined by $\varphi(z)=S^{n}$ for any $z \in S^{n}$ is admissible and $\varphi^{*}$ is an infinite set. $\varphi^{*} \ni 0$ means that $\left(p^{*}\right)^{-1} q^{*}=0$ for some selected pair $(p, q)$ of $\varphi$ and $\varphi^{*} \not \supset 0$ means that $\left(p^{*}\right)^{-1} q^{*} \neq 0$ for any selected pair $(p, q)$ of $\varphi$.

Theorem 3.10. Let $X$ be an ANR space and $\varphi: X \rightarrow X$ a compact admissible mapping. If $L\left(\varphi^{*}\right)$ contains a non-trivial element, there exists a fixed point $x_{0} \in X$, that is, $x_{0} \in \varphi\left(x_{0}\right)$.

Proof. We can choose a selected pair $(p, q)$ where a Vietoris mapping $p$ : $\Gamma \rightarrow X$ and a compact mapping $q: \Gamma \rightarrow X$. We may assume $L\left(\left(p^{*}\right)^{-1} q^{*}\right) \neq 0$. By Theorem 3.9, there exists a coincidence point $z \in \Gamma$ such that $p(z)=q(z)$. Since it holds $x \in q p^{-1}(x) \subset \varphi(x)$ where $x=p(z)$, we obtain the result.

From Theorem 3.8, we easily obtain a fixed point theorem for a compact weak admissible mappings $\varphi: U \rightarrow U$ where $U$ is an open set of a normed space. Note that our result is broader than L. Górniewicz's result, because we works in the category of paracompact Hausdorff spaces.

\section{$\S 4$. Equivariant Fundamental Cohomology Class}

To begin with, we review some results of M. Nakaoka $[13,14,15,16]$ for notation and later applications. We must study his papers carefully, because he discuss his theory in the category of manifolds and use the singular (co)homology theory. 
Let $X$ be a Hausdorff space with an involution $T . X^{2}=X \times X$ has the involution $T$ of the switching mapping $T(x, y)=(y, x)$. We use the notation $G^{2}=G \otimes G$ for an abelian group $G$. Let $\pi$ be the group of order 2. A $\pi$-space means a topological space with an involution $T$. Let $A$ be a subspace of a $\pi$-space $X$ invariant under the action. For such a pair $(X, A)$, the equivariant cohomology group $\bar{H}_{\pi}^{*}\left(X, A ; \mathbf{F}_{2}\right)$ and the equivariant homology group $\bar{H}_{*}^{\pi}\left(X, A ; \mathbf{F}_{2}\right)$ are defined by $\bar{H}^{*}\left(S^{\infty} \times_{\pi} X, S^{\infty} \times_{\pi} A ; \mathbf{F}_{2}\right)$ and $\bar{H}_{*}\left(S^{\infty} \times_{\pi} X, S^{\infty} \times_{\pi} A ; \mathbf{F}_{2}\right)$ respectively. Hereafter we shall use (co)homology theory with coefficient in the prime field $\mathbf{F}_{2}$ of order 2. We sometimes abbreviate the coefficient $\mathbf{F}_{2}$ in the (co)homology theory, when the expression is complicated.

M. Nakaoka proved the Steenrod isomorphism of finite type (cf. $\S 3$ in [13]) for the singular (co)homology theory. We can prove the Steenrod isomorphism for the Alexander-Spanier (co)homology theory. $W$ is the chain complex with generators $e_{i}, T e_{i}(i=0,1, \ldots)$ and boundaries $\partial_{i+1}\left(e_{i+1}\right)=$ $e_{i}+(-1)^{i+1} T e_{i}(i=0,1, \ldots)$.

Theorem 4.1. Let $X$ be a compact ANR space $X$ of finite type.

$$
\begin{aligned}
& \bar{H}_{*}\left(S^{\infty} \times_{\pi} X^{2} ; \mathbf{F}_{2}\right) \cong H_{*}\left(W \otimes_{\pi} \bar{H}_{*}\left(X ; \mathbf{F}_{2}\right)^{2} ; \mathbf{F}_{2}\right) \\
& \bar{H}^{*}\left(S^{\infty} \times_{\pi} X^{2} ; \mathbf{F}_{2}\right) \cong H^{*}\left(\operatorname{Hom}_{\pi}\left(W, \bar{H}^{*}\left(X ; \mathbf{F}_{2}\right)^{2}\right) ; \mathbf{F}_{2}\right)
\end{aligned}
$$

Proof. Let $\mathcal{U}=\left\{U_{\lambda}, T U_{\lambda} \mid \lambda \in \Lambda\right\}$ be an open covering of $S^{\infty}$ such that $U_{\lambda} \cap T U_{\lambda}=\emptyset$. If a covering $\mathcal{V}$ of $X$ with a free involution $T$ satisfies $V \cap T V=\emptyset$ for $V \in \mathcal{V}, \mathcal{V}$ is called a proper covering of $X$. Let $\mathcal{V}$ and $\mathcal{W}$ be open coverings of $X$ and $Y$ respectively. $\mathcal{V} \times \mathcal{W}=\{V \times W \mid V \in \mathcal{V}, W \in \mathcal{W}\}$ is an open covering of $X \times Y$. Set $\mathcal{V}^{2}=\mathcal{V} \times \mathcal{V}$. Note that $p\left(\mathcal{U} \times \mathcal{V}^{2}\right)=\left\{p\left(U_{\lambda} \times V \times V^{\prime}\right) \mid U_{\lambda} \in \mathcal{U}, V, V^{\prime} \in\right.$ $\mathcal{V}\}$ is a family of evenly covered open sets of the projection $p: E \times X^{2} \rightarrow E \times_{\pi} X^{2}$ (cf. Chapter 2 in [17]).

We can prove the following chain equivalence corresponding to Theorem 1 in [13]:

$$
\rho: C_{*}\left(\left(S^{\infty} \times X^{2}\right)\left(\mathcal{U} \times \mathcal{V}^{2}\right)\right) \rightarrow W \otimes C_{*}(X(\mathcal{V}))^{2}
$$

where $\mathcal{U}$ is a proper open covering of $S^{\infty}$ and $\mathcal{V}$ is an open covering of $X$. The proof proceeds exactly same as the case of $S_{*}\left(S^{\infty} \times X^{2}\right)$. Hereafter we often use the corresponding notation of [13]. There exists a chain equivalence (cf. Theorem 2 in [13]):

$$
\Pi: C_{*}\left(\left(S^{\infty} \times X^{2}\right)\left(\mathcal{U} \times \mathcal{V}^{2}\right)\right) / \pi \rightarrow C_{*}\left(\left(S^{\infty} \times{ }_{\pi} X^{2}\right)\left(p\left(\mathcal{U} \times \mathcal{V}^{2}\right)\right)\right) .
$$


By using $\rho$ and $\Pi$, we obtain

$$
\chi: H_{*}\left(W \otimes_{\pi} C_{*}(X(\mathcal{V}))^{2}\right) \cong H_{*}\left(\left(S^{\infty} \times_{\pi} X^{2}\right)\left(p\left(\mathcal{U} \times \mathcal{V}^{2}\right)\right)\right) .
$$

By making use of the isomorphism $\xi_{*}\left(\eta_{*}\right)^{-1}: H_{*}\left(W \otimes_{\pi} H_{*}(X(\mathcal{V}))^{2}\right) \cong H_{*}\left(W \otimes_{\pi}\right.$ $\left.C_{*}(X(\mathcal{V}))^{2}\right)($ cf. $\S 2$ in $[13])$, we obtain

$$
\chi \xi_{*}\left(\eta_{*}\right)^{-1}: H_{*}\left(W \otimes_{\pi} H_{*}(X(\mathcal{V}))^{2}\right) \cong H_{*}\left(\left(S^{\infty} \times_{\pi} X^{2}\right)\left(p\left(\mathcal{U} \times \mathcal{V}^{2}\right)\right)\right) .
$$

Therefore we obtain the result corresponding to [13] for a compact ANR space of finite type by taking the inverse limits. Here we used Lemma 2.3 for each dimension.

By making use of the isomorphism $\xi_{*}\left(\eta_{*}\right)^{-1}: H^{*}\left(\operatorname{Hom}_{\pi}\left(W, H^{*}\left(C^{*}(X(\mathcal{V}))\right)^{2}\right)\right.$ $\rightarrow H^{*}\left(\operatorname{Hom}_{\pi}\left(W, C^{*}(X(\mathcal{V}))^{2}\right)\right)$ and the isomorphism $\chi^{*} \nu \mu_{*}$ :

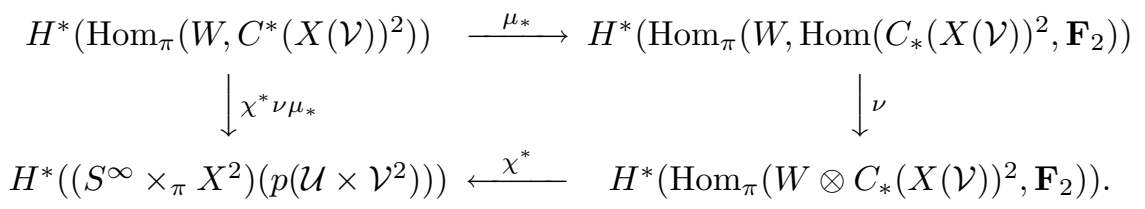

we have the isomorphism between $H^{*}\left(\operatorname{Hom}_{\pi}\left(W, H^{*}\left(C^{*}(X(\mathcal{V}))\right)^{2}\right)\right.$ and $H^{*}\left(\left(S^{\infty} \times_{\pi} X^{2}\right)\left(p\left(\mathcal{U} \times \mathcal{V}^{2}\right)\right)\right)$. Therefore we obtain the second isomorphism by taking the direct limits.

If you wish to avoid the calculation of inverse limits, you may apply the result of the singular (co)homology theory for ANR spaces of finite type $H^{*}(X ; \mathbf{F})$ (cf. Theorem 6.9 .1 of [17]). When $X$ is a compact ANR space, we obtain the following theorem (cf. Theorem 4 in M. Nakaoka [13]). Though it is proved for more general space, it is sufficient for our purpose.

Theorem 4.2. Let $X$ be a compact $A N R$ space of finite type. If $\left\{a_{j} \mid j \in\right.$ $J\}$ is an ordered basis of $\bar{H}_{*}\left(X ; \mathbf{F}_{2}\right)$, then $\left\{P_{i}\left(a_{j}\right), P\left(a_{j}, a_{k}\right) \mid i \geqq 0, j, k \in\right.$ $J, j<k\}$ is a basis of $\bar{H}_{*}\left(S^{\infty} \times_{\pi} X^{2} ; \mathbf{F}_{2}\right)$. If $\left\{\alpha_{j} \mid j \in J\right\}$ is an ordered basis of $\bar{H}^{*}\left(X ; \mathbf{F}_{2}\right)$, then $\left\{P_{i}\left(\alpha_{j}\right), P\left(\alpha_{j}, \alpha_{k}\right) \mid i \geqq 0, j, k \in J, j<k\right\}$ is a basis of $\bar{H}^{*}\left(S^{\infty} \times_{\pi} X^{2} ; \mathbf{F}_{2}\right)$. If $\left\{a_{j} \mid j \in J\right\}$ and $\left\{\alpha_{j} \mid j \in J\right\}$ are dual basis, then $\left\{P_{i}\left(a_{j}\right), P\left(a_{j}, a_{k}\right)\right\}$ and $\left\{P_{i}\left(\alpha_{j}\right), P\left(\alpha_{j}, \alpha_{k}\right)\right\}$ are also dual.

We can define cohomology operations $P_{i}(\alpha)$ and $P(\alpha, \beta)$ of the AlexanderSpanier cohomology theory by using Theorem 4.1 as M. Nakaoka [13]. Though the naturality of $P_{i}(-)$ with respect to a mapping is clear for compact ANR spaces of finite type, it is not so clear for general spaces. Because the Steenrod 
isomorphisms do not hold for general spaces. Therefore we must exactly inspect the cohomology operations $P_{i}(\alpha)$ and $P(\alpha, \beta)$ of the Alexander-Spanier cohomology theory for a paracompact Hausdorff space $X$. The cohomology operation

$$
P_{i}: H^{*}\left(X(\mathcal{V}) ; \mathbf{F}_{2}\right) \rightarrow H^{*}\left(\operatorname{Hom}_{\pi}\left(W, H^{*}\left(C^{*}(X(\mathcal{V})) ; \mathbf{F}_{2}\right)^{2}\right)\right.
$$

is defined by $P_{i}(\alpha)=u_{i}(\alpha), u_{i}(\alpha)\left(e_{i}\right)=\alpha \otimes \alpha, u_{j}(\alpha)\left(e_{i}\right)=0(i \neq j)$.

By making use of $P_{i}(\alpha)$ and the morphism $\chi^{*} \nu \mu_{*} \xi_{*}\left(\eta_{*}\right)^{-1}$ (cf. [13]) and taking the limits we can define the cohomology operation

$$
P_{i}: \bar{H}^{*}\left(X ; \mathbf{F}_{2}\right) \rightarrow \bar{H}^{*}\left(S^{\infty} \times_{\pi} X^{2} ; \mathbf{F}_{2}\right) .
$$

From the definition, we can easily verify the naturality of the operation $P_{i}(\alpha)$ with respect mappings i.e. $P\left(f^{*}(\alpha)\right)=\left(1 \times_{\pi} f^{2}\right)^{*}(P(\alpha))$ where $f: X \rightarrow Y$ and $\alpha \in \bar{H}^{*}\left(Y ; \mathbf{F}_{2}\right)$.

$P(\alpha, \beta)$ is similarly defined. Since $P(\alpha, \beta)$ is the same as $\phi^{*}(\alpha \times \beta)$ where $\phi^{*}$ is the transfer mapping, $\phi^{*}(\alpha \times \beta)$ is defined for general spaces. We obtain the naturality of $P(\alpha, \beta)$ with respect to mappings, i.e. $\phi^{*}\left(1 \times f^{2}\right)^{*}(1 \times \alpha \times \beta)=$ $\left(1 \times_{\pi} f^{2}\right)^{*} \phi^{*}(1 \times \alpha \times \beta)$ where $f: X \rightarrow Y$ and $\alpha, \beta \in \bar{H}^{*}\left(Y ; \mathbf{F}_{2}\right)$.

Now we remark the transfer mapping for the Alexander-Spanier cohomology theory. In the case of the singular cohomology theory, the Gysin-Smith exact sequence of a double covering space $p: \tilde{X} \rightarrow X$ is defined by the following sequence:

$$
0 \rightarrow S^{*}(X) \stackrel{p^{*}}{\rightarrow} S^{*}(\tilde{X}) \stackrel{\phi^{*}}{\rightarrow} S^{*}(X) \rightarrow 0
$$

where $\phi^{*}$ is the transfer mapping and $p^{*}$ is defined by the projection $p$. By using the Vietoris complex, we can obtain the similar result for the Alexander-Spanier cohomology theory.

Since the transfer homomorphism $\phi^{*}$ is also defined by using Thom isomorphism of a fiber bundle, we may use the Thom-Gysin sequence instead of the Gysin-Smith sequence. Now we explain the Thom isomorphism for the Alexander-Spanier cohomology theory. Let $E$ be the mapping cylinder of a double covering $p: \tilde{X} \rightarrow X$ and $\tilde{p}: E \rightarrow X$ the associated projection. According to Theorem 6.9 .14 in [17] and the notation there, we have the isomorphism:

$$
\Psi: \bar{H}^{q}\left(X ; \mathbf{F}_{2}\right) \cong \bar{H}^{q+1}\left(E, \tilde{X} ; \mathbf{F}_{2}\right) .
$$

We can define the transfer homomorphism $\phi^{*}: \bar{H}^{i}\left(\tilde{X} ; \mathbf{F}_{2}\right) \rightarrow \bar{H}^{i}\left(X ; \mathbf{F}_{2}\right)$ by $\Psi^{-1} \delta$ where $\delta$ is the coboundary homomorphism of the cohomology exact sequence of the pair $(E, \tilde{X})$. 
From the cohomology exact sequence of a pair $(E, \tilde{X})$ and Thom isomorphism, we obtain the following exact sequence:

$$
\bar{H}^{i-1}\left(\tilde{X} ; \mathbf{F}_{2}\right) \stackrel{\phi^{*}}{\rightarrow} \bar{H}^{i-1}\left(X ; \mathbf{F}_{2}\right) \stackrel{\nu}{\rightarrow} \bar{H}^{i}\left(X ; \mathbf{F}_{2}\right) \stackrel{p^{*}}{\rightarrow} \bar{H}^{i}\left(\tilde{X} ; \mathbf{F}_{2}\right) \stackrel{\phi^{*}}{\rightarrow} \bar{H}^{i}\left(X ; \mathbf{F}_{2}\right)
$$

where $\nu: \bar{H}^{i-1}\left(X ; \mathbf{F}_{2}\right) \rightarrow \bar{H}^{i}\left(X ; \mathbf{F}_{2}\right)$ is defined by $\left(\tilde{p}^{*}\right)^{-1} j^{*} \Psi$. Thom-Gysin sequence is natural for mappings between double covering spaces. We prepare a lemma for later application.

Lemma 4.3. Let $p_{i}: \tilde{X}_{i} \rightarrow X_{i}(i=1,2)$ be double covering spaces over paracompact Hausdorff spaces and $\tilde{f}: \tilde{X}_{1} \rightarrow \tilde{X}_{2}$ and $f: X_{1} \rightarrow X_{2}$ be continuous mappings such that $p_{2} \tilde{f}=f p_{1}$. $\tilde{f}$ induces a (co)homology isomorphism if and only if $f$ induces a (co)homology isomorphism.

Proof. From the Gysin-Smith sequence (4.5), we have the following commutative diagram:

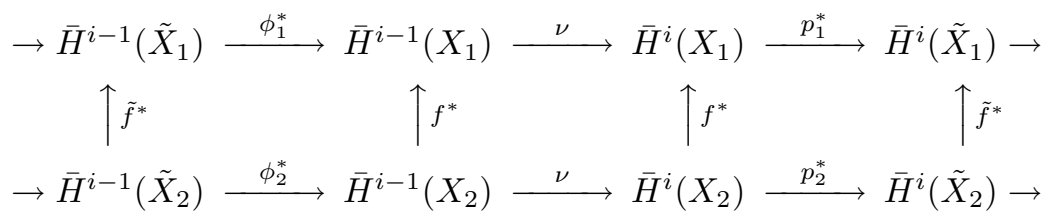

Assume that $\tilde{f}^{*}$ is an isomorphism. Firstly we can prove the injectivity of $f^{*}$ by the induction on $i$ in the above diagram and secondly the surjectivity of $f^{*}$ by the induction on $i$. Since the verification is easy, we omit the detail. The converse is true by the five lemma.

A connected metric space $X$ is said to be an $n$-dimensional Poincaré space, if there exist a class $\mu \in \bar{H}^{n}\left(X ; \mathbf{F}_{2}\right)$ and an isomorphism:

$$
(-) \cap \mu: \bar{H}^{q}\left(X ; \mathbf{F}_{2}\right) \rightarrow \bar{H}_{n-q}\left(X ; \mathbf{F}_{2}\right) .
$$

Especially we easily see $\bar{H}^{n}\left(X ; \mathbf{F}_{2}\right)=\mathbf{F}_{2}, \bar{H}_{n}\left(X ; \mathbf{F}_{2}\right)=\mathbf{F}_{2}$ and $\bar{H}^{q}\left(X ; \mathbf{F}_{2}\right)=$ $0, \bar{H}_{q}\left(X ; \mathbf{F}_{2}\right)=0$ for $q>n$. If there exists a continuous mapping $f: X \rightarrow Y$ where $Y$ is a closed manifold and $f$ induces a homology isomorphism $f_{*}$ : $\bar{H}_{*}\left(X ; \mathbf{F}_{2}\right) \rightarrow \bar{H}_{*}\left(Y ; \mathbf{F}_{2}\right)$, then $X$ is a Poincaré space by $f_{*}\left(f^{*}(\alpha) \cap \mu\right)=\alpha \cap$ $f_{*}(\mu)$. Similarly if there exists a continuous mapping $g: Y \rightarrow X$ where $Y$ is a closed manifold and $g$ induces a homology isomorphism $g_{*}: \bar{H}_{*}\left(Y ; \mathbf{F}_{2}\right) \rightarrow$ $\bar{H}_{*}\left(X ; \mathbf{F}_{2}\right)$, then $X$ is a Poincaré space. Note that the orbit space $X_{\pi}$ of a metric space $X$ is also a metric space by Smirnov metrization theorem (cf. Theorem 4.4.19 in [3]). 
Though $S^{n} \times S^{\infty}$ is a Poincaré space with the free involution $T(x, y)=$ $(x,-y)$, the orbit space $S^{n} \times R P^{\infty}$ is not a Poincaré space. If the covering dimension of a paracompact Hausdorff space $X$ with a free involution is a finite, $\left\{p \mid \bar{H}^{p}\left(X_{\pi} ; \mathbf{F}_{2}\right) \neq 0\right\}$ is bounded. Let $\mathcal{U}$ be an open covering of $X_{\pi}$ where any open set of $\mathcal{U}$ is evenly covered by $p$. We take a proper refinement $\mathcal{V}$ of $p^{-1}(\mathcal{U})$ such that the intersection of any $n+2$ open sets of $\mathcal{V}$ is empty for $n=\operatorname{dim} X$. The covering $p(\mathcal{V})$ shows the finiteness of the covering dimension of $X_{\pi}$. The following lemma is useful for our application.

Lemma 4.4. Let $X$ be a Poincaré space with a free involution. If $\left\{p \mid \bar{H}^{p}\left(X_{\pi} ; \mathbf{F}_{2}\right) \neq 0\right\}$ is bounded, then the orbit space $X_{\pi}$ is a Poincaré space.

Proof. Let $\mu \in \bar{H}_{n}\left(X ; \mathbf{F}_{2}\right)$ be the class which induces the isomorphism (4.6). There exists the element $\mu^{\prime} \in \bar{H}_{n}\left(X_{\pi} ; \mathbf{F}_{2}\right)$ such that $\phi_{*}\left(\mu^{\prime}\right)=\mu$ by the Gysin-Smith homology sequence and the hypothesis on $\left\{p \mid \bar{H}^{p}\left(X ; \mathbf{F}_{2}\right) \neq 0\right\}$. Moreover we see $\bar{H}_{n}\left(X_{\pi} ; \mathbf{F}_{2}\right)=\mathbf{F}_{2}$ and $\bar{H}_{i}\left(X_{\pi} ; \mathbf{F}_{2}\right)=0$ for $i>n$ by considering the Gysin-Smith exact sequence. We have the ladder of the Gysin-Smith exact sequences:

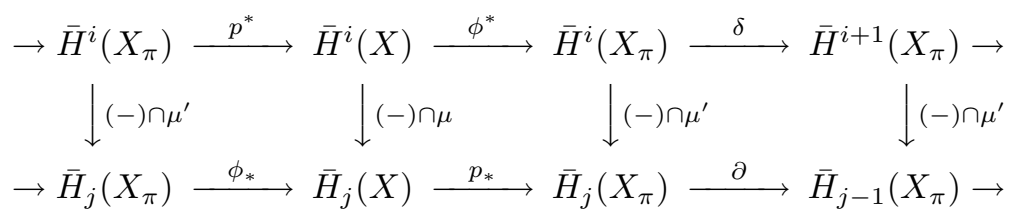

where $i+j=n$. The commutativity is proved by $p^{*}(\alpha) \cap \phi_{*}\left(\mu^{\prime}\right)=\phi_{*}\left(\alpha \cap \mu^{\prime}\right)$ etc. where $\alpha \in \bar{H}^{*}\left(X_{\pi} ; \mathbf{F}_{2}\right)$. By the induction from the lower dimension of cohomology theory, we can prove the injectivity of $(-) \cap \mu^{\prime}$. From this and the induction, we obtain also the surjectivity of $(-) \cap \mu^{\prime}$.

Let $M$ be an $m$-dimensional closed manifold with an involution $T$. Let $\Delta: M \rightarrow M^{2}$ be defined by $\Delta(x)=(x, T(x))$. When $T$ is trivial, $\Delta$ is the ordinary diagonal. $\Delta M$ is the invariant subspace under the action. M. Nakaoka defined the equivariant fundamental class of $\Delta M$ in $M^{2}$ denoted by $\hat{U}_{M} \in \bar{H}_{\pi}^{m}\left(M^{2}, M^{2}-\Delta M ; \mathbf{F}_{2}\right)$ (cf. $\S 2$ in [16]). The image of $\hat{U}_{M}$ in $\bar{H}_{\pi}^{m}\left(M^{2} ; \mathbf{F}_{2}\right)$ is denoted by $\hat{U}_{M}^{\prime}$, and is called the equivariant diagonal cohomology class of $M$.

The involution $T$ on $M^{2}$ is given by $T\left(x, x^{\prime}\right)=\left(x^{\prime}, x\right)$. Therefore $\Delta$ is an equivariant mapping. Hereafter, we sometimes use the same notation for involutions, if there is not confusion. Note that the involution $\tilde{T}$ on $S^{\infty} \times{ }_{\pi} M^{2}$ is given by $\tilde{T}\left(x, y, y^{\prime}\right)=\left(T x, y^{\prime}, y\right)$. For a paracompact Hausdorff space $N$ with 
a free involution $T$, there exists an equivariant mapping $h: N \rightarrow S^{\infty}$. We also define the element:

$$
\hat{U}_{N, M} \in \bar{H}^{m}\left(N \times_{\pi}\left(M^{2}, M^{2}-\Delta M\right) ; \mathbf{F}_{2}\right)
$$

by $\hat{U}_{N, M}=\left(h \times_{\pi} i d_{M^{2}}\right)^{*}\left(\hat{U}_{M}\right)$ where $h \times_{\pi} i d_{M^{2}}: N \times_{\pi}\left(M^{2}, M^{2}-\Delta(M)\right) \rightarrow$ $S^{\infty} \times_{\pi}\left(M^{2}, M^{2}-\Delta(M)\right)$. Note that $N \times_{\pi}\left(M^{2}, M^{2}-\Delta M\right)$ is a pair of paracompact Hausdorff spaces by Theorem 5.1.33 and $\S 5.5 .5$ in [3]. Set

$$
\Delta_{N}=j^{*}\left(\hat{U}_{N, M}\right) \in \bar{H}^{m}\left(N \times_{\pi} M^{2} ; \mathbf{F}_{2}\right)
$$

where $j: N \times_{\pi} M^{2} \rightarrow N \times_{\pi}\left(M^{2}, M^{2}-\Delta M\right)$. In the case of $N=S^{\infty}$, we have $\hat{U}_{N, M}=\hat{U}_{M}$ and $\Delta_{N}=\hat{U}_{M}^{\prime}$. Set $\Delta_{\infty}=\hat{U}_{M}^{\prime}$ for $N=S^{\infty}$. Clearly, we have $\left(h \times_{\pi} 1\right)^{*}\left(\Delta_{\infty}\right)=\Delta_{N}$.

The following theorem is essentially proved in Proposition 1.3 in [14]. Though he states his result for a manifold $N$, it holds for a paracompact Hausdorff space $N$. For $f: N \rightarrow M$, define $\hat{f}: N \rightarrow N \times M^{2}$ by $\hat{f}(x)=$ $(x, f(x), f(T(x)))$ which is $\pi$-equivariant. Hence we have $\hat{f}_{\pi}: N_{\pi} \rightarrow N \times_{\pi} M^{2}$.

Theorem 4.5. $\quad$ Let $N$ be a paracompact Hausdorff space with a free involution $T$ and $M$ a closed manifold with an involution $T^{\prime}$. If a continuous mapping $f: N \rightarrow M$ satisfies $\hat{f}_{\pi}^{*}\left(\Delta_{N}\right) \neq 0$, the set $A(f)=\{x \in N \mid f(T(x))=$ $\left.T^{\prime}(f(x))\right\}$ is not empty.

Proof. Consider the following diagram:

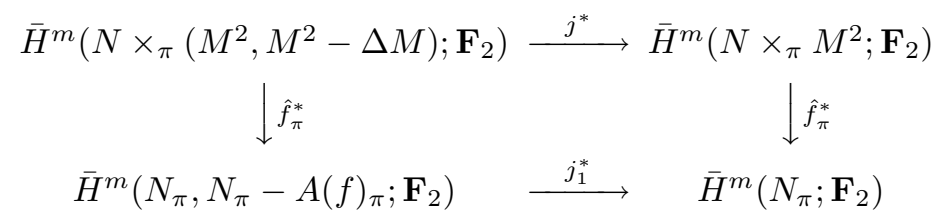

where $A(f)_{\pi}$ is the orbit space of $A(f)$. Since the class $\Delta_{N} \in \bar{H}^{m}\left(N \times_{\pi}\right.$ $\left.M^{2} ; \mathbf{F}_{2}\right)$ is the image of $\hat{U}_{N, M} \in \bar{H}^{m}\left(N \times_{\pi}\left(M^{2}, M^{2}-\Delta M\right) ; \mathbf{F}_{2}\right)$, we obtain $\hat{f}_{\pi}^{*}\left(\hat{U}_{N, M}\right) \neq 0$ from the condition $\hat{f}_{\pi}^{*}\left(\Delta_{N}\right) \neq 0$. Therefore we have $\bar{H}^{m}\left(N_{\pi}, N_{\pi}-A(f)_{\pi} ; \mathbf{F}_{2}\right) \neq 0$. Finally, we obtain $A(f)_{\pi} \neq \emptyset$ and $A(f) \neq \emptyset$.

We can obtain the detailed result of the above theorem under the restriction of a source space.

Theorem 4.6. Under the condition of Theorem 4.5, if $N$ is an $n$ dimensional Poincaré space and the covering dimension of $N$ is finite, it holds $\operatorname{dim} A(f) \geqq n-m$. 
Proof. In the diagram of Theorem 4.5, the class $f_{\pi}^{*}\left(\Delta_{N}\right) \in \bar{H}^{m}\left(N_{\pi} ; \mathbf{F}_{2}\right)$ is not zero. Since $N_{\pi}$ is a Poincaré space, there exists a class $\alpha \in \bar{H}^{n-m}\left(N_{\pi} ; \mathbf{F}_{2}\right)$ such that $f_{\pi}^{*}\left(\Delta_{N}\right) \cup \alpha \neq 0$. If $i^{*}(\alpha)=0$ where $i: A(f)_{\pi} \rightarrow N_{\pi}$, we see $i_{V}^{*}(\alpha)=0$ by the continuity of the Alexander-Spanier cohomology theory where $V$ is an open neighborhood of $A(f)_{\pi}$ in $N_{\pi}$ and $i_{V}: V \rightarrow N_{\pi}$ is the inclusion. Therefore there is a class $\beta \in \bar{H}^{n-m}\left(N_{\pi}, V ; \mathbf{F}_{2}\right)$ such that $j_{2}^{*}(\beta)=\alpha$ where $j_{2}^{*}: \bar{H}^{n-m}\left(N_{\pi}, V ; \mathbf{F}_{2}\right) \rightarrow \bar{H}^{n-m}\left(N_{\pi} ; \mathbf{F}_{2}\right)$.

Since $\hat{f}_{\pi}^{*}\left(\Delta_{N}\right)$ is the image of $\hat{f}_{\pi}^{*}\left(\hat{U}_{N, M}\right)$ under $j_{1}^{*}$, it holds

$$
j^{*}\left(\hat{f}_{\pi}^{*}\left(\hat{U}_{N, M}\right) \cup \beta\right)=j_{1}^{*}\left(\hat{f}_{\pi}^{*}\left(\hat{U}_{N, M}\right)\right) \cup j_{2}^{*}(\beta)=\hat{f}_{\pi}^{*}\left(\Delta_{N}\right) \cup \alpha .
$$

The left hand side is zero by $\hat{f}_{\pi}^{*}\left(\hat{U}_{N, M}\right) \cup \beta=0$ in $\bar{H}^{*}\left(N_{\pi}, V \cup\left(N_{\pi}-A(f)_{\pi}\right) ; \mathbf{F}_{2}\right)$ $=0$. Here we used that $V \cup\left(N_{\pi}-A(f)_{\pi}\right)=N_{\pi}$. The right hand side is not zero by our hypothesis. From the contradiction, we see $i^{*}(\alpha) \neq 0 \in$ $\bar{H}^{n-m}\left(A(f)_{\pi} ; \mathbf{F}_{2}\right)$.

If it holds $\bar{H}^{k}\left(A(f) ; \mathbf{F}_{2}\right)=0$ for all $k \geqq n-m$, we see $\bar{H}^{k}\left(A(f)_{\pi} ; \mathbf{F}_{2}\right) \neq 0$ for all $k \geqq n-m$ by making use of the Gysin-Smith sequence of $p: A(f) \rightarrow$ $A(f)_{\pi}$. This contradict that the covering dimension of $N$ is finite. Therefore we see $\bar{H}^{k}\left(A(f) ; \mathbf{F}_{2}\right) \neq 0$ for some $k \geqq n-m$.

\section{$\S 5 . \quad$ The Equivariant Lefschetz Class}

M. Nakaoka determined $\Delta_{\infty}$ and $\Delta_{N}$ in Proposition 3.2 in [14]. Though he describe some statements under the assumption that $N$ is a manifold in his papers, we can easily generalize to the case that $N$ is a paracompact Hausdorff space. The following theorem is given in Theorem 2.3 in [16].

Theorem 5.1. Let $M$ be an m-dimensional closed manifold with an involution T. Assume that

$$
i^{*}=0: \bar{H}^{q}\left(M ; \mathbf{F}_{2}\right) \rightarrow \bar{H}^{q}\left(M^{\pi} ; \mathbf{F}_{2}\right) \quad\left(q \geqq \frac{m}{2}\right)
$$

where $M^{\pi}$ is the fixed point set of the involution $T$. Then, for a basis $\left\{\alpha_{1}, \ldots\right.$, $\left.\alpha_{s}\right\}$ of $\bar{H}^{*}\left(M ; \mathbf{F}_{2}\right)$, we have

$$
\Delta_{\infty}=\sum_{i<j} c_{i j} \phi^{*}\left(1 \times \alpha_{i} \times \alpha_{j}\right)
$$

where $\phi^{*}: \bar{H}^{*}\left(S^{\infty} \times M^{2} ; \mathbf{F}_{2}\right) \rightarrow \bar{H}^{*}\left(S^{\infty} \times_{\pi} M^{2} ; \mathbf{F}_{2}\right)$ is the transfer mapping and $C=\left(c_{i j}\right)$ is the inverse of the matrix $Y=\left(y_{i j}\right)$ with $\left.y_{i j}=<\alpha_{i} \cup T^{*} \alpha_{j},[M]\right\rangle$. 
For a closed manifold $M$ with an involution $T$, we define a bilinear form $\circ: \bar{H}^{*}\left(M ; \mathbf{F}_{2}\right) \otimes \bar{H}^{*}\left(M ; \mathbf{F}_{2}\right) \rightarrow \mathbf{F}_{2}$ by

$$
\alpha \circ \beta=<\alpha \cup T^{*} \beta,[M]>.
$$

The bilinear form $\circ$ is called a symplectic form, if it satisfies $\alpha \circ \alpha=0$ for any $\alpha \in \bar{H}^{*}\left(M ; \mathbf{F}_{2}\right)$.

Under the condition

$$
i^{*}=0: \bar{H}^{\frac{m}{2}}\left(M ; \mathbf{F}_{2}\right) \rightarrow \bar{H}^{\frac{m}{2}}\left(M^{\pi} ; \mathbf{F}_{2}\right) \quad(m: \text { even })
$$

the bilinear form $\circ$ is symplectic form by Proposition 5.5 in [16]. According to linear algebra, we can find a basis $\left\{\mu_{1}, \ldots, \mu_{s}, \mu_{1}^{\prime}, \ldots, \mu_{s}^{\prime}\right\}$ of $\bar{H}^{*}\left(M ; \mathbf{F}_{2}\right)$ called a symplectic basis such that

$$
\mu_{i} \circ \mu_{j}=0, \quad \mu_{i}^{\prime} \circ \mu_{j}^{\prime}=0, \quad \mu_{i} \circ \mu_{j}^{\prime}=\delta_{i j} .
$$

The following theorem is easily deduced from Theorem 5.1 (cf. Theorem 3.4 in [14]).

Theorem 5.2. Under the condition and the notation of Theorem 5.1, let $\left\{\mu_{1}, \ldots, \mu_{s}, \mu_{1}^{\prime}, \ldots, \mu_{s}^{\prime}\right\}$ be a symplectic basis of $\bar{H}^{*}\left(M ; \mathbf{F}_{2}\right)$. Then we have

$$
\Delta_{\infty}=\sum_{i=1}^{s} \phi^{*}\left(1 \times \mu_{i} \times \mu_{i}^{\prime}\right)
$$

where $\phi^{*}$ is the transfer mapping. A similar formula holds for $\Delta_{N} \in \bar{H}^{*}\left(N \times_{\pi}\right.$ $\left.M^{2} ; \mathbf{F}_{2}\right)$ for a paracompact Hausdorff space $N$.

Definition 5.1. $\quad$ Let $N$ be a paracompact Hausdorff space with a free involution $T$ and $M$ a closed manifold with an involution $T^{\prime}$. For a continuous mapping $f: N \rightarrow M$, we define the equivariant Lefschetz class of $f$ by

$$
\hat{f}_{\pi}^{*}\left(\Delta_{N}\right) \in \bar{H}^{m}\left(N_{\pi} ; \mathbf{F}_{2}\right) .
$$

The following formula is calculated in the proof of Proposition 6.4 in [16] (cf. Theorem 5.2 in [14]).

Theorem 5.3. Let $N$ be a paracompact Hausdorff space with a free involution $T$ and $M$ a closed manifold with an involution $T^{\prime}$ satisfying the condition (5.1) and $\left\{\mu_{1}, \ldots, \mu_{s}, \mu_{1}^{\prime}, \ldots, \mu_{s}^{\prime}\right\}$ a symplectic basis of $\bar{H}^{*}\left(M ; \mathbf{F}_{2}\right)$. For a continuous mapping $f: N \rightarrow M$, we have

$$
\hat{f}_{\pi}^{*}\left(\Delta_{N}\right)=\sum_{i=1}^{s} \phi^{*}\left(f^{*}\left(\mu_{i}\right) \cup T^{*} f^{*}\left(\mu_{i}^{\prime}\right)\right)
$$

where $\phi^{*}: \bar{H}^{*}\left(N ; \mathbf{F}_{2}\right) \rightarrow \bar{H}^{*}\left(N_{\pi} ; \mathbf{F}_{2}\right)$ is the transfer mapping. 
Definition 5.2. $\quad$ Let $N$ be a paracompact Hausdorff space with a free involution $T$ and $M$ an $m$-dimensional closed topological manifolds with an involution $T^{\prime}$ and $\left\{\mu_{1}, \ldots, \mu_{s}, \mu_{1}^{\prime}, \ldots, \mu_{s}^{\prime}\right\}$ a symplectic basis of $\bar{H}^{*}\left(M ; \mathbf{F}_{2}\right)$. The Lefschetz class $\Delta_{N, \varphi}$ of an admissible mapping $\varphi: N \rightarrow M$ is defined by the following set:

$$
\Delta_{N, \varphi}=\left\{\sum_{i=1}^{s} \phi^{*}\left(\left(p^{*}\right)^{-1} q^{*}\left(\mu_{i}\right) \cup T^{*}\left(p^{*}\right)^{-1} q^{*}\left(\mu_{i}^{\prime}\right)\right)\right\}
$$

where $(p, q)$ is any selected pair of $\varphi$. For $\left[N_{\pi, \alpha}\right] \in \bar{H}^{m}\left(N_{\pi} ; \mathbf{F}_{2}\right)$, the equivariant Lefschetz number $\mathcal{L}\left(\varphi:\left[N_{\pi, \alpha}\right]\right)$ is defined by the following set:

$$
\mathcal{L}\left(\varphi:\left[N_{\pi, \alpha}\right]\right)=\left\{\sum_{i=1}^{s}<\phi^{*}\left(\left(p^{*}\right)^{-1} q^{*}\left(\mu_{i}\right) \cup T^{*}\left(p^{*}\right)^{-1} q^{*}\left(\mu_{i}^{\prime}\right)\right),\left[N_{\pi, \alpha}\right]>\right\}
$$

where $(p, q)$ is any selected pair of $\varphi$. For single valued mapping $f$, we use $L\left(f:\left[N_{\pi, \alpha}\right]\right)$ instead of $\mathcal{L}\left(\varphi:\left[N_{\pi, \alpha}\right]\right)$.

When $N$ and $M$ are $m$-dimensional closed manifolds, M. Nakaoka defined the equivariant point index $I_{\Pi}(f)$ by $<\hat{f}_{\pi}^{*}\left(\Delta_{\infty}\right),[N / \Pi]>$ and the Lefschetz number $\lambda_{\Pi}(f)$ by $\sum_{i=1}^{r}<f^{*}\left(\mu_{i}\right) \cup T^{*} f^{*}\left(\mu_{i}^{\prime}\right),[N]>$ (cf. $\S 1$ and $\S 5$ in [14], [16]). For the case of single-valued mapping and $N_{\pi, \alpha}=N_{\pi}$, these two numbers coincide each other (cf. Theorem 7.1 in [16]). And it holds also for an $m$-dimensional Poincaré space $N$ with a free involution and a finite covering dimension. Therefore our definition of the equivariant Lefschetz number is a natural generalization.

Let $N$ be a paracompact Hausdorff space with a free involution $T$. Consider the following diagram:

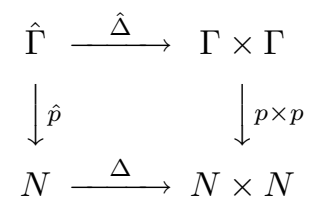

where $\Delta$ is given by $\Delta(x)=(x, T(x)) . \hat{\Gamma}$ is defined by $\left\{\left(y, y^{\prime}\right) \in \Gamma \times \Gamma \mid p(y)=\right.$ $\left.T\left(p\left(y^{\prime}\right)\right)\right\} . \quad \hat{p}$ is defined by $\hat{p}\left(y, y^{\prime}\right)=p(y)$ and $\hat{\Delta}$ is the natural inclusion. Clearly the above square is a pull-back square. Involutions on $N^{2}, \Gamma^{2}$ are given by switching mappings $T\left(x, x^{\prime}\right)=\left(x^{\prime}, x\right)$. All mappings are $\pi$-equivariant with respect to their involutions. Note that $\hat{\Gamma}$ has the free involution $\hat{T}$. The following lemma is a key to our purposes. The notation in the next lemma is the same in the diagram (5.10). 
Lemma 5.4. Let $N$ be a paracompact Hausdorff space with a free involution $T$ and $p: \Gamma \rightarrow N$ be a Vietoris mapping. Then $\hat{p}: \hat{\Gamma} \rightarrow N$ is a $\pi$-equivariant Vietoris mapping and $\hat{\Gamma}$ is a paracompact Hausdorff space. $\hat{p}_{\pi}: \hat{\Gamma}_{\pi} \rightarrow N_{\pi}$ is a Vietoris mapping and $\hat{\Gamma}_{\pi}$ is a paracompact Hausdorff space. Moreover if $N$ is a metric space and $A$ is a $\pi$-invariant closed subspace of $N$, then $\bar{H}^{*}\left(\hat{\Gamma}-\hat{p}^{-1}(A) ; \mathbf{F}_{2}\right)$ and $\bar{H}^{*}\left(\hat{\Gamma}_{\pi}-\hat{p}_{\pi}^{-1}\left(A_{\pi}\right) ; \mathbf{F}_{2}\right)$ are isomorphic to $\bar{H}^{*}\left(N-A ; \mathbf{F}_{2}\right)$ and $\bar{H}^{*}\left(N_{\pi}-A_{\pi} ; \mathbf{F}_{2}\right)$ respectively.

Proof. Since $p$ is proper, we easily see that $p \times p$ is also proper. Because we find compact sets $K_{1}, K_{2}$ in $N$ for a compact set $K$ of $N \times N$ such that $K \subset K_{1} \times K_{2}$. We easily see that $(p \times p)^{-1}(K)$ is compact. Similarly $\hat{p}: \hat{\Gamma} \rightarrow N$ is proper. Since $N$ is a paracompact Hausdorff space and $\hat{p}$ is proper, $\hat{\Gamma}$ is also a paracompact Hausdorff space by Theorem 4 in [9] or Theorem 5.1.35 in [3]. We can easily prove the acyclicity of $(p \times p)^{-1}\left(x, x^{\prime}\right)$ by using Exercise E of Chapter 6 in [17]. Therefore $\hat{p}: \hat{\Gamma} \rightarrow N$ is a $\pi$-equivariant Vietoris mapping. Therefore $\bar{H}^{*}(\hat{\Gamma})$ is isomorphic to $\bar{H}^{*}(N) . N_{\pi}$ and $\hat{\Gamma}_{\pi}$ are paracompact Hausdorff spaces by Theorem 5.1.33 in [3]. Since a fiber $\hat{p}^{-1}(x)$ of $\hat{p}: \hat{\Gamma} \rightarrow N$ is homeomorphic to a fiber $\hat{p}_{\pi}^{-1}([x])$ of $\hat{p}_{\pi}: \hat{\Gamma}_{\pi} \rightarrow N_{\pi}, \hat{p}_{\pi}$ is a Vietoris mapping. Therefore we obtain the first statement.

For the last statement, since $N-A$ is an open set, $\hat{p}: \hat{\Gamma}-\hat{p}^{-1}(A) \rightarrow N-A$ is also onto proper mapping. Since $N$ is a metric space, $N-A$ is a paracompact Hausdorff space and $\hat{\Gamma}-\hat{p}^{-1}(A)$ is also a paracompact Hausdorff space by Theorem 4 in [9]. By Vietoris's Theorem 3.1, $\hat{p}^{*}$ is an isomorphism. Similarly we obtain the result for $\hat{p}_{\pi}: \hat{\Gamma}_{\pi}-\hat{p}_{\pi}^{-1}\left(A_{\pi}\right) \rightarrow N_{\pi}-A_{\pi}$ by Lemma 4.3.

In the second statement of the above theorem, we have to assume that $N$ is a metric space, because a subset of a paracompact Hausdorff space is not necessarily a paracompact space.

Theorem 5.5. Let $N$ be a paracompact Hausdorff space with a free involution $T$ and $M$ an $m$-dimensional closed topological manifold with an involution $T^{\prime}$ satisfying the condition (5.1). For an admissible mapping $\varphi: N \rightarrow M$, if $\mathcal{L}\left(\varphi:\left[N_{\pi, \alpha}\right]\right)$ contains a non-trivial element, then there exists a point $x_{0} \in N$ such that $T^{\prime} \varphi\left(x_{0}\right) \cap \varphi\left(T\left(x_{0}\right)\right) \neq \emptyset$. Moreover if $N$ is an $n$-dimensional Poincaré space and the covering dimension of $N$ is finite, it holds $\operatorname{dim} A(\varphi) \geqq n-m$ where $A(\varphi)=\left\{x \in N \mid T^{\prime}(\varphi(x)) \cap \varphi(T(x)) \neq \emptyset\right\}$.

Proof. We use the notation in the diagram (5.10). Let $(p, q)$ be a selected 
pair of $\varphi$. To begin with, consider the following diagram.

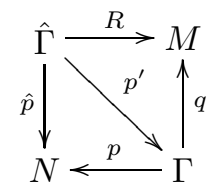

where $p^{\prime}\left(y, y^{\prime}\right)=y$ and $R\left(y, y^{\prime}\right)=q(y)$.

Define

$$
\begin{aligned}
A(R) & =\left\{\left(y, y^{\prime}\right) \in \hat{\Gamma} \mid T^{\prime} R\left(y, y^{\prime}\right)=R\left(T\left(y, y^{\prime}\right)\right)\right\} \\
& =\left\{\left(y, y^{\prime}\right) \in \hat{\Gamma} \mid T^{\prime} q(y)=q\left(y^{\prime}\right)\right\} .
\end{aligned}
$$

Clearly we see $A(R) \subset(\hat{p})^{-1}(A(\varphi))$. The space $A(\varphi)$ is a closed set of $N$ by the upper semi-continuity of $\varphi$.

Since the mapping $\hat{p}: \hat{\Gamma} \rightarrow N$ is $\pi$-equivariant, $\left(\hat{p}_{\pi}\right)_{*}$ is isomorphic by Lemma 5.4. We can find $\left[\hat{N}_{\pi, \alpha}\right] \in \bar{H}_{*}\left(\hat{\Gamma}_{\pi} ; \mathbf{F}_{2}\right)$ such that $\left(\hat{p}_{\pi}\right)_{*}\left[\hat{N}_{\pi, \alpha}\right]=\left[N_{\pi, \alpha}\right]$. Since $\varphi$ is admissible, we remark $\varphi^{*} \ni\left(p^{*}\right)^{-1} q^{*} . \hat{R}: \hat{\Gamma} \rightarrow \hat{\Gamma} \times M^{2}$ is defined by $\hat{R}\left(y, y^{\prime}\right)=\left(y, y^{\prime}, q(y), q\left(y^{\prime}\right)\right)$. In the following, we shall prove $\left(\hat{R}_{\pi}\right)^{*}\left(\Delta_{\hat{\Gamma}}\right) \neq 0$ by showing $L\left(R:\left[\hat{N}_{\pi, \alpha}\right]\right)=<\left(\hat{R}_{\pi}\right)^{*}\left(\Delta_{\hat{\Gamma}}\right),\left[\hat{N}_{\pi, \alpha}\right]>\neq 0$ (cf. Theorem 5.3).

Let $\left\{\mu_{1}, \ldots, \mu_{s}, \mu_{1}^{\prime}, \ldots, \mu_{s}^{\prime}\right\}$ be a symplectic basis of $\bar{H}^{*}\left(M ; \mathbf{F}_{2}\right)$. We have the formula:

$$
\left(\hat{R}_{\pi}\right)^{*}\left(\Delta_{\hat{\Gamma}}\right) \in\left(\hat{p}_{\pi}\right)^{*} \Delta_{N, \varphi}
$$

by the following calculation:

$$
\begin{aligned}
\left(\hat{R}_{\pi}\right)^{*}\left(\Delta_{\hat{\Gamma}}\right) & =\sum_{i=1}^{s} \phi^{*}\left(R^{*}\left(\mu_{i}\right) \cup \hat{T}^{*} R^{*}\left(\mu_{i}^{\prime}\right)\right) \\
& =\sum_{i=1}^{s} \phi^{*}\left(\hat{p}^{*}\left(p^{*}\right)^{-1} q^{*}\left(\mu_{i}\right) \cup \hat{T}^{*} \hat{p}^{*}\left(p^{*}\right)^{-1} q^{*}\left(\mu_{i}^{\prime}\right)\right) \\
& =\sum_{i=1}^{s} \phi^{*}\left(\hat{p}^{*}\left(p^{*}\right)^{-1} q^{*}\left(\mu_{i}\right) \cup \hat{p}^{*} T^{*}\left(p^{*}\right)^{-1} q^{*}\left(\mu_{i}^{\prime}\right)\right) \\
& =\hat{p}_{\pi}^{*}\left(\sum_{i=1}^{s} \phi^{*}\left(\left(p^{*}\right)^{-1} q^{*}\left(\mu_{i}\right) \cup T^{*}\left(p^{*}\right)^{-1} q^{*}\left(\mu_{i}^{\prime}\right)\right)\right) \\
& \in \hat{p}_{\pi}^{*}\left(\Delta_{N, \varphi}\right) .
\end{aligned}
$$

And also we have the formula: 


$$
\begin{aligned}
L\left(R:\left[\hat{N}_{\pi, \alpha}\right]\right) & =<\left(\hat{R}_{\pi}\right)^{*}\left(\Delta_{\hat{\Gamma}}\right),\left[\hat{N}_{\pi, \alpha}\right]> \\
& \in<\left(\hat{p}_{\pi}\right)^{*} \Delta_{N, \varphi},\left[\hat{N}_{\pi, \alpha}\right]> \\
& =<\Delta_{N, \varphi},\left[N_{\pi, \alpha}\right]> \\
& =\mathcal{L}\left(\varphi:\left[N_{\pi, \alpha}\right]\right) .
\end{aligned}
$$

From this conclusion and our assumption, we have $\hat{R}_{\pi}^{*}\left(\Delta_{\hat{\Gamma}}\right) \neq 0$. Therefore we obtain an equivariant point $\left(y_{0}, y_{0}^{\prime}\right) \in \hat{\Gamma}$ such that

$$
T^{\prime}\left(R\left(y_{0}, y_{0}^{\prime}\right)\right)=R\left(\hat{T}\left(y_{0}, y_{0}^{\prime}\right)\right)
$$

by Theorem 4.5. This show $T^{\prime} q\left(y_{0}\right)=q\left(y_{0}^{\prime}\right)$. Therefore we see $q\left(y_{0}\right) \in$ $\varphi\left(x_{0}\right), T^{\prime} q\left(y_{0}\right) \in \varphi\left(T\left(x_{0}\right)\right)$ where $x_{0}=p\left(y_{0}\right)$, that is, $T^{\prime}\left(\varphi\left(x_{0}\right)\right) \cap \varphi\left(T\left(x_{0}\right)\right) \neq \emptyset$.

For the proof of the second part, consider the following diagram:

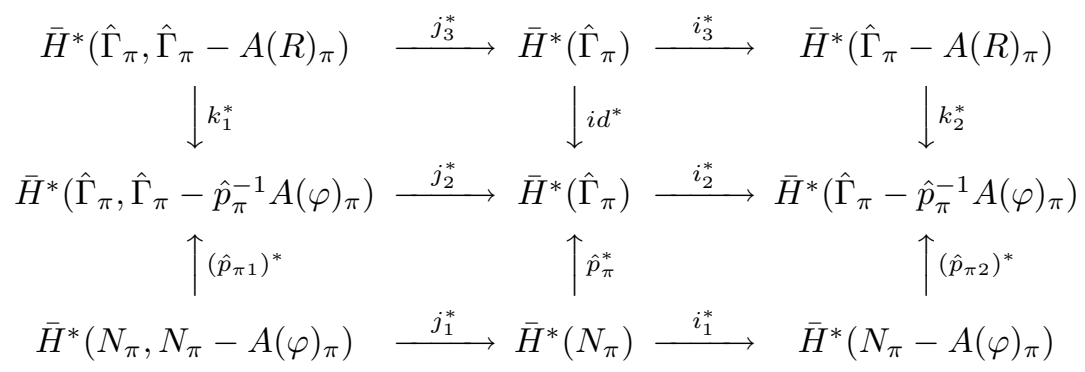

where $k_{1}, k_{2}$ are induced by natural inclusions and $\hat{p}_{\pi 1}, \hat{p}_{\pi 2}$ are induced by $\hat{p}_{\pi}$. Spaces $\hat{\Gamma}-A(R), \hat{\Gamma}-\hat{p}^{-1} A(\varphi)$ and $N-A(\varphi)$ have natural involutions induced by $\hat{T}$ and $T$ respectively.

Since $\left(\hat{R}_{\pi}\right)^{*}\left(\Delta_{\hat{\Gamma}}\right)$ is the image of the class $\left(\hat{R}_{\pi}\right)^{*}\left(\Delta_{\hat{\Gamma}, M}\right)$ in $H^{*}\left(\hat{\Gamma}_{\pi}, \hat{\Gamma}_{\pi}-\right.$ $\left.A(R)_{\pi} ; \mathbf{F}_{2}\right)$ under $j_{3}^{*}$ by Theorem $4.5, i_{2}^{*}\left(\hat{R}_{\pi}\right)^{*}\left(\Delta_{\hat{\Gamma}}\right)$ is zero. By the definition of $\Delta_{N, \varphi}$ and the formula (5.12), we have $\left(\hat{R}_{\pi}\right)^{*}\left(\Delta_{\hat{\Gamma}}\right)=\hat{p}_{\pi}^{*}(\alpha)$ for a non zero element $\alpha \in \Delta_{N, \varphi}$. We see $\hat{p}_{\pi 2}^{*} i_{1}^{*}(\alpha)=i_{2}^{*} \hat{p}_{\pi}^{*}(\alpha)=0$ in the above diagram. Because of bijectivity of $\left(\hat{p}_{\pi 2}\right)^{*}$ by Lemma 5.4 , we obtain $i_{1}^{*}(\alpha)=0$. Therefore we have $\bar{H}^{m}\left(N_{\pi}, N_{\pi}-A(\varphi)_{\pi} ; \mathbf{F}_{2}\right) \neq 0$. We obtain the last statement by applying Theorem 4.6 and its proof.

In the above theorem, if we take the fundamental cycle $\left[N_{\pi}\right]$, we obtain a generalization of Theorem 5.3 of M. Nakaoka [14] to an admissible mapping, where the source space is a Poincare space with a finite covering dimension (cf. Theorem 7.1 in [16]). We obtain the following corollary as the special case. 
Corollary 5.6. Let $N$ be an n-dimensional Poincaré space with a free involution $T$ which has a finite covering dimension and $M$ a closed topological manifold with a non-trivial involution $T^{\prime}$ which has the homology groups of the $n$-dimensional sphere. For an admissible mapping $\varphi: N \rightarrow M$, if $\varphi^{*}$ contains a non-trivial element, then there exists a point $x_{0} \in N$ such that $T^{\prime} \varphi\left(x_{0}\right) \cap \varphi\left(T\left(x_{0}\right)\right) \neq \emptyset$.

Proof. Since $T^{\prime}$ is a non-trivial involution, we easily see $\bar{H}^{n}\left(M^{\pi} ; \mathbf{F}_{2}\right)=0$ by Poincaré duality. Hence $M$ satisfies the condition (5.1). A symplectic basis on $M$ is given by $\{1, \mu\}$ where $\mu$ is a dual element of $[M]$. By taking a selected pair $(p, q)$, we have $\left(p^{*}\right)^{-1} q^{*}(\mu)=\nu$ where $\nu$ is a dual element of $[N]$. We see

$$
<\phi^{*}\left(\left(p^{*}\right)^{-1} q^{*}(1) \cup T^{*}\left(\left(p^{*}\right)^{-1} q^{*}(\mu)\right)\right),\left[N_{\pi}\right]>=1 \in \mathcal{L}\left(\varphi:\left[N_{\pi}\right]\right)
$$

by Definition 5.2. Therefore we obtain the result by Theorem 5.5.

We must remark the case that $N$ is an $n$-dimensional Poincaré space with a free involution $T$ which has the homology groups of the $n$-dimensional sphere and $M$ is an $n$-dimensional closed topological manifold with an involution $T^{\prime}$ satisfying the condition (5.1). When $\varphi^{*}$ contains a non-trivial element, we assert that $M$ is an $n$-dimensional homology sphere. Let $\left\{\mu_{1}, \ldots, \mu_{s}, \mu_{1}^{\prime}, \ldots, \mu_{s}^{\prime}\right\}$ be a symplectic basis on $M$ where $\mu_{1}=1$ and $\mu_{1}^{\prime}$ is a dual element of [M]. If $s \geqq 2$, it holds $\mu_{i} T^{*} \mu_{i}^{\prime}=\mu_{1}^{\prime}$ for $i \geqq 2$ by the definition of a symplectic basis. By the dimensional reason, we see $\left(p^{*}\right)^{-1} q^{*}\left(\mu_{i} T^{*} \mu_{i}^{\prime}\right)=0$ for $i \geqq 2$ and therefore $\left(p^{*}\right)^{-1} q^{*}\left(\mu_{1}^{\prime}\right)=0$. By the non-triviality of $\varphi^{*}$, we have $\left(p^{*}\right)^{-1} q^{*}\left(\mu_{1}^{\prime}\right) \neq 0$. From the contradiction, we obtain $s=1$. This means that $M$ is an $n$-dimensional homology sphere. Therefore we can not obtain any new result.

\section{§6. Borsuk-Ulam Theorem for Set-Valued Mappings}

Let $M$ be an $m$-dimensional closed topological manifold with a trivial involution. The $i$-th Wu class $v_{i} \in \bar{H}^{i}\left(M ; \mathbf{F}_{2}\right)(i=0,1,2, \ldots)$ of a manifold $M$ is defined by $\left\langle S q^{i} \alpha,[M]>=<\alpha \cup v_{i},[M]>\right.$, especially $v_{0}=1$ and $v_{i}=0$ for $i>\frac{m}{2}$.

When the involution of $M$ is trivial, M. Nakaoka determined the class $\Delta_{\infty}$ by Theorem 3.5 in [15] and Theorem 9.1 in [16]. Note $P=P_{0}$.

Theorem 6.1. Let $M$ be an m-dimensional closed topological manifold. Let $\left\{\alpha_{1}, \ldots, \alpha_{s}\right\}$ be a basis for $\bar{H}^{*}\left(M ; \mathbf{F}_{2}\right)$. Set

$$
d_{*}([M])=\sum_{j, k} \eta_{j k} a_{j} \times a_{k} \quad\left(\eta_{j k} \in \mathbf{F}_{2}\right)
$$


where $d(x)=(x, x)$ and $a_{i}=\alpha_{i} \cap[M]$. Then, it holds

$$
\Delta_{\infty}=\sum_{i \geqq 0} \omega^{m-2 i} P\left(v_{i}\right)+\sum_{j<k}\left(\eta_{j k}+\eta_{j j} \eta_{k k}\right) \phi^{*}\left(1 \times \alpha_{j} \times \alpha_{k}\right)
$$

where $\omega=c\left(S^{\infty} \times M^{2}, T\right)$ and $v_{i}=v_{i}(M)$ the $i$-th $W u$ class of $M$ and $\phi^{*}$ : $\bar{H}^{*}\left(S^{\infty} \times M^{2} ; \mathbf{F}_{2}\right) \rightarrow \bar{H}^{*}\left(S^{\infty} \times{ }_{\pi} M^{2} ; \mathbf{F}_{2}\right)$ is the transfer homomorphism.

Though the above theorem is proved by using the singular cohomology theory, we can easily verify the same statement for the Alexander-Spanier cohomology theory.

If a space $X$ has a free involution, we have the isomorphism $q_{\pi}^{*}: \bar{H}^{k}\left(X_{\pi} ; \mathbf{F}_{2}\right)$ $\cong \bar{H}^{k}\left(S^{\infty} \times_{\pi} X ; \mathbf{F}_{2}\right)$ by Lemma 4.3. Define $Q: \bar{H}^{k}\left(X ; \mathbf{F}_{2}\right) \rightarrow \bar{H}^{2 k}\left(X_{\pi} ; \mathbf{F}_{2}\right)$ by

$$
Q(\alpha)=\left(q_{\pi}^{*}\right)^{-1}\left(1 \times_{\pi} \Delta\right)^{*} P_{0}(\alpha)
$$

where $\Delta(x)=(x, T x)$ (cf. $\S 2$ in [15]). From the formula (6.1), we obtain the following theorem (cf. Theorem 3.5 in [15], Proposition 9.2 in [16]).

Theorem 6.2. $\quad$ Under the notation of Theorem 6.1 , let $N$ be a paracompact Hausdorff space with a free involution $T$ and $f: N \rightarrow M$ a continuous mapping. Then it holds

$$
\hat{f}_{\pi}^{*}\left(\Delta_{N}\right)=\sum_{i \geqq 0} c^{m-2 i} Q\left(f^{*} v_{i}\right)+\sum_{j<k}\left(\eta_{j k}+\eta_{j j} \eta_{k k}\right) \phi^{*}\left(f^{*}\left(\alpha_{j}\right) \cup T^{*} f^{*}\left(\alpha_{k}\right)\right)
$$

where $c=c(N, T)$ and $\phi^{*}: \bar{H}^{*}\left(N ; \mathbf{F}_{2}\right) \rightarrow \bar{H}^{*}\left(N_{\pi} ; \mathbf{F}_{2}\right)$ is the transfer homomorphism

Proof. Though the theorem is proved for the singular cohomology theory in [15], we can easily verify the same statement for the Alexander-Spanier cohomology theory. For the purpose, we have verified in $\S 4$ the commutativities $P\left(f^{*}(\alpha)\right)=\left(1 \times_{\pi} f^{2}\right)^{*}(P(\alpha))$ and $\phi^{*}\left(1 \times f^{2}\right)^{*}(1 \times \alpha \times \beta)=\left(1 \times_{\pi} f^{2}\right)^{*} \phi^{*}(1 \times \alpha \times \beta)$ where $f: N \rightarrow M$ and $\alpha, \beta \in \bar{H}^{*}\left(M ; \mathbf{F}_{2}\right)$. Apply $\left(q_{\pi}^{*}\right)^{-1}\left(1 \times_{\pi} f^{2}\right)^{*}\left(1 \times_{\pi} \Delta\right)^{*}$ for the formula of Theorem 6.1. From the above naturalities and the isomorphism $q_{\pi}^{*}: \bar{H}^{*}\left(S^{\infty} \times_{\pi} N ; \mathbf{F}_{2}\right) \rightarrow \bar{H}^{*}\left(N_{\pi} ; \mathbf{F}_{2}\right)$, we easily obtain our formula.

L. Górniewicz studied the Borsuk-Ulam theorem for set-valued mapping in $\S 37$ and $\S 43$ of [7]. He proved it for an upper semi-continuous acyclic mapping $\varphi: M \rightarrow \mathbf{R}^{n}$ where $M$ is a closed topological manifold with the homology 
groups of the $n$-dimensional sphere. In this paper, we shall prove a generalization of the Borsuk-Ulam theorem for the class of admissible mappings which contains upper semi-continuous acyclic mappings (cf. $[5,6]$ ). Now we shall prove our main theorem. The proof proceeds by the same method as the proof of Theorem 5.5.

Theorem 6.3. $\quad$ Let $N$ be a paracompact Hausdorff space with a free involution $T$ and $M$ an $m$-dimensional closed topological manifold. Assume that the first Stiefel-Whitney class $c(N, T)$ satisfies $c(N, T)^{m} \neq 0$. If a set-valued mapping $\varphi: N \rightarrow M$ is admissible and $\varphi^{*}$ contains the trivial element, then there exists a point $x_{0} \in N$ such that $\varphi\left(x_{0}\right) \cap \varphi\left(T\left(x_{0}\right)\right) \neq \emptyset$. Moreover if $N$ is an $n$-dimensional Poincaré space and the covering dimension of $N$ is finite, it holds $\operatorname{dim} A(\varphi) \geqq n-m$ where $A(\varphi)=\{x \in N \mid \varphi(x) \cap \varphi(T(x)) \neq \emptyset\}$.

Proof. To begin with, we choose a selected pair $(p, q)$ of $\varphi$ where $p$ : $\Gamma \rightarrow N, q: \Gamma \rightarrow M$. By our hypothesis, $p$ and $q$ satisfy $\left(p^{*}\right)^{-1} q^{*}=0$ for positive dimensions. Now consider the diagram defined by (5.11). We see $R^{*}=0$ for positive dimensions by the condition $\left(p^{*}\right)^{-1} q^{*}=0$. Since $\hat{p}_{\pi}^{*}$ is an isomorphism by Lemma 5.4, we have $\hat{p}_{\pi}^{*}\left(c^{m}\right)=\hat{c}^{m} \neq 0$ in $\bar{H}^{*}\left(\hat{\Gamma}_{\pi} ; \mathbf{F}_{2}\right)$ where $\hat{c}=c(\hat{\Gamma}, \hat{T}), c=c(N, T)$ and $\hat{c}=\hat{p}_{\pi}^{*}(c)$.

Now we calculate $\left(\hat{R}_{\pi}\right)^{*}\left(\Delta_{\hat{\Gamma}}\right)$ by Theorem 6.2 . Since we have $\phi^{*}\left(R^{*}\left(\alpha_{j}\right) \cup\right.$ $\left.T^{*} R^{*}\left(\alpha_{k}\right)\right)=0$ and $\hat{c}^{m-2 i} Q\left(R^{*}\left(v_{i}\right)\right)=0$ for $i>0$ from our hypothesis, we obtain

$$
\left(\hat{R}_{\pi}\right)^{*}\left(\Delta_{\hat{\Gamma}}\right)=\hat{c}^{m} Q\left(R^{*}\left(v_{0}\right)\right)=\hat{c}^{m} \neq 0
$$

from the formula (6.4) in Theorem 6.2. We conclude $A(R) \neq \emptyset$ by Theorem 4.5 where $A(R)=\left\{\left(y, y^{\prime}\right) \in \hat{\Gamma} \mid R\left(y, y^{\prime}\right)=R\left(y^{\prime}, y\right)\right\}$. Since it holds $A(R) \subset$ $\hat{p}^{-1} A(\varphi)$, we obtain the first statement.

For the second statement, since spaces $\hat{\Gamma}-A(R), \hat{\Gamma}-\hat{p}^{-1} A(\varphi)$ and $N-A(\varphi)$ have natural involutions induced by $\hat{T}$ and $T$ respectively, we have their orbit spaces $\hat{\Gamma}_{\pi}-A(R)_{\pi}, \hat{\Gamma}_{\pi}-\hat{p}_{\pi}^{-1} A(\varphi)_{\pi}$ and $N_{\pi}-A(\varphi)_{\pi}$. Now we shall use the second diagram defined in the proof of Theorem 5.5 and the notation there. Since $A(\varphi)$ is a $\pi$-invariant closed subset of $N$, we have the isomorphism $\left(\hat{p}_{\pi 2}\right)^{*}$ : $\bar{H}^{*}\left(N_{\pi}-A(\varphi)_{\pi} ; \mathbf{F}_{2}\right) \rightarrow \bar{H}^{*}\left(\hat{\Gamma}_{\pi}-\hat{p}_{\pi}^{-1}\left(A(\hat{\varphi})_{\pi}\right) ; \mathbf{F}_{2}\right)$ by Lemma 5.4. Note that $\left(\hat{R}_{\pi}\right)^{*}\left(\Delta_{\hat{\Gamma}}\right)=\hat{c}^{m}$ is the image of $c^{m} \in \bar{H}^{*}\left(N_{\pi} ; \mathbf{F}_{2}\right)$, that is, $\left(\hat{p}_{\pi}\right)^{*}\left(c^{m}\right)=\hat{c}^{m}$. Since $\hat{c}^{m}$ is the image of $\left(\hat{R}_{\pi}\right)^{*}\left(\hat{U}_{\hat{\Gamma}, M}\right)$ under $j_{3}^{*}$, it holds $i_{2}^{*}\left(\hat{c}^{m}\right)=0$. From this, we see $\left(\hat{p}_{\pi 2}\right)^{*}\left(i_{1}\right)^{*}\left(c^{m}\right)=\left(i_{2}\right)^{*}\left(\hat{p}_{\pi}\right)^{*}\left(c^{m}\right)=\left(i_{2}\right)^{*}\left(\hat{c}^{m}\right)=0$ in the diagram and therefore $\left(i_{1}\right)^{*}\left(c^{m}\right)=0$ because of the bijectivity of $\left(\hat{p}_{\pi 2}\right)^{*}$. If $\bar{H}^{m}\left(N_{\pi}, N_{\pi}-\right.$ $\left.A(\varphi)_{\pi} ; \mathbf{F}_{2}\right)=0$, we obtain $c^{m}=0$ which contradicts $c^{m} \neq 0$. Therefore we 
see $\bar{H}^{m}\left(N_{\pi}, N_{\pi}-A(\varphi)_{\pi} ; \mathbf{F}_{2}\right) \neq 0$. We obtain the last statement by applying Theorem 4.6 and its proof.

If $N$ is a paracompact Hausdorff space with a free involution $T$ which has the homology groups of the $n$-dimensional sphere, the cohomology ring $\bar{H}^{*}\left(N_{\pi} ; \mathbf{F}_{2}\right)$ of $N_{\pi}$ is a truncated polynomial ring $\mathbf{F}_{2}[c] /\left(c^{n+1}\right)$ from the GysinSmith sequence of double covering space, where $c=c(N, T)$ is the generator of $\bar{H}^{1}\left(N_{\pi} ; \mathbf{F}_{2}\right)$. If $\varphi^{*}$ contains the trivial element, we easily obtain the following corollary from Theorem 6.3.

Corollary 6.4. Let $N$ be a paracompact Hausdorff space with a free involution $T$ which has the homology groups of the $n$-dimensional sphere. Let $M$ be an $m$-dimensional closed topological manifold with $m \leqq n$. If a set-valued mapping $\varphi: N \rightarrow M$ is admissible and $\varphi^{*}$ contains the trivial element, then there exists a point $x_{0} \in N$ such that $\varphi\left(x_{0}\right) \cap \varphi\left(T\left(x_{0}\right)\right) \neq \emptyset$. Moreover if $N$ is an $n$-dimensional Poincaré space with a finite covering dimension, it holds $\operatorname{dim} A(\varphi) \geqq n-m$.

When $M$ is a closed topological manifold which has the homology groups of the $m$-dimensional sphere. The homology groups of $M^{\prime}=M-\{a\}$ is trivial for positive dimensions. If it holds $\varphi(N) \neq M$, we see $R(\hat{\Gamma}) \neq M$ and $R^{*}=0$ for positive dimensions. Therefore we obtain the following result from Theorem 6.3 .

Corollary 6.5. Let $N$ be a paracompact Hausdorff space with a free involution $T$. Let $M$ be a closed topological manifold which has the homology groups of the m-dimensional sphere. Assume that the first Stiefel-Whitney class $c(N, T)$ satisfies $c(N, T)^{m} \neq 0$ and a set-valued mapping $\varphi: N \rightarrow M$ satisfies $\varphi(N) \neq M$. If a set-valued mapping $\varphi$ is admissible, then there exists a point $x_{0} \in N$ such that $\varphi\left(x_{0}\right) \cap \varphi\left(T\left(x_{0}\right)\right) \neq \emptyset$. Moreover if $N$ is an $n$-dimensional Poincaré space with a finite covering dimension, it holds $\operatorname{dim} A(\varphi) \geqq n-m$.

Let $X$ be a space with a free involution $T$ and $S^{k}$ the $k$-dimensional sphere with the antipodal involution. Define $\gamma(X)$ and $\operatorname{Ind}(X)$ by

$$
\begin{aligned}
\gamma(X) & =\inf \left\{k \mid f: X \rightarrow S^{k} \text { equivariant mapping }\right\} \\
\operatorname{Ind}(X) & =\sup \left\{k \mid c^{k} \neq 0\right\}
\end{aligned}
$$

respectively, where $c \in \bar{H}^{1}\left(X_{\pi} ; \mathbf{F}_{2}\right)$ is the class $c=f_{\pi}^{*}(\omega)$ for an equivariant mapping $f: X \rightarrow S^{\infty}$. If $X$ is a compact space with a free involution, it holds the following formula (cf. $\S 3$ in [4]):

$$
\operatorname{Ind}(X) \leqq \gamma(X) \leqq \operatorname{dim} X \text {. }
$$


K. Gęba and L. Górniewicz determined $\operatorname{Ind} A(\varphi)$ of an admissible mapping $\varphi: S^{n+k} \rightarrow \mathbf{R}^{n}$ in [4]. We shall generalize their result.

Corollary 6.6. Let $N$ be a paracompact Hausdorff space with a free involution $T$ which satisfies $c(N, T)^{n} \neq 0$. Let $M$ be an $m$-dimensional closed topological manifold with $m \leqq n$. If a set-valued mapping $\varphi: N \rightarrow M$ is admissible and $\varphi^{*}$ contains the trivial element, it holds $\operatorname{Ind} A(\varphi) \geqq n-m$.

Proof. Consider the second diagram in the proof of Theorem 5.5. We can find a class $\alpha \in \bar{H}^{m}\left(N_{\pi}, N_{\pi}-A(\varphi)_{\pi} ; \mathbf{F}_{2}\right)$ such that $j_{1}^{*}(\alpha)=c^{m}(c=c(N, T))$ where $j_{1}^{*}: \bar{H}^{m}\left(N_{\pi}, N_{\pi}-A(\varphi)_{\pi} ; \mathbf{F}_{2}\right) \rightarrow \bar{H}^{m}\left(N_{\pi} ; \mathbf{F}_{2}\right)$. If $i^{*}\left(c^{n-m}\right)$ is equal to 0 where $i: A(\varphi)_{\pi} \rightarrow N_{\pi}$, there is an open neighborhood $V$ of $A(\varphi)_{\pi}$ such that $i_{V}^{*}\left(c^{n-m}\right)=0$ and $i_{V}: V \rightarrow N_{\pi}$. Then we can find $\beta \in \bar{H}^{m}\left(N_{\pi}, V ; \mathbf{F}_{2}\right)$ such that $j_{2}^{*}(\beta)=c^{n-m}$ where $j_{2}^{*}: \bar{H}^{m}\left(N_{\pi}, V ; \mathbf{F}_{2}\right) \rightarrow \bar{H}^{m}\left(N_{\pi} ; \mathbf{F}_{2}\right)$. It holds the following equation:

$$
j^{*}(\alpha \cup \beta)=j_{1}^{*}(\alpha) \cup j_{2}^{*}(\beta)=c^{m} \cup c^{n-m}=c^{n} .
$$

The left hand side is zero by $\alpha \cup \beta=0$ in $\bar{H}^{*}\left(N_{\pi},\left(N_{\pi}-A(f)_{\pi}\right) \cup V ; \mathbf{F}_{2}\right)=0$. Note that $\left(N_{\pi}-A(f)_{\pi}\right) \cup V=N_{\pi}$. The right hand side is not zero by our hypothesis $c^{n} \neq 0$. From the contradiction, we see $i^{*}\left(c^{n-m}\right) \neq 0 \in$ $\bar{H}^{n-m}\left(A(\varphi)_{\pi} ; \mathbf{F}_{2}\right)$. Therefore we obtain the result by $i^{*}(c)^{n-m}=i^{*}\left(c^{n-m}\right)$.

A similar result for a single-valued mapping is proved in [18] under some condition. His method uses the (co)homology theory with compact support and geometric consideration. Since our method is algebraic, the proof is more simple and general. When $N$ is a closed topological manifold with a free involution $T$ which has the homology groups of the $n$-dimensional sphere, we can prove the above theorem by considering the following commutative diagram (cf. Chapter $6 \S 1$ in E.H. Spanier [17]):

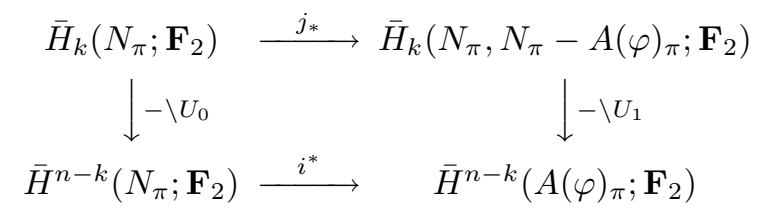

where $U_{0}, U_{1}$ are the restrictions of the fundamental cocycle $U \in \bar{H}^{n}\left(X^{2}, X^{2}-\right.$ $\left.d(X) ; \mathbf{F}_{2}\right)$ respectively.

From Theorem 6.3 and Corollary 6.5, we can easily obtain the following corollary. We can also obtain a generalization of Theorem 43.10 in $\mathrm{L}$. Górniewicz [7] and Theorem 2.7 in [4] by considering $\mathbf{R}^{m}$ as the subspace of the $m$-dimensional sphere $S^{m}$. 
Corollary 6.7. $\quad$ Let $N$ be a closed topological manifold with a free involution $T$ which has the homology groups of the $n$-dimensional sphere. Let $M$ be an m-dimensional closed topological manifold with $m \leqq n$. Assume that a set-valued mapping $\varphi: N \rightarrow M$ satisfies $\varphi(N) \neq M$. If a set-valued mapping $\varphi$ is admissible, then there exists a point $x_{0} \in N$ such that $\varphi\left(x_{0}\right) \cap \varphi\left(T\left(x_{0}\right)\right) \neq \emptyset$. Moreover it holds $\operatorname{Ind} A(\varphi) \geqq n-m$.

\section{Acknowledgement}

The author would like to thank the referee for careful reading of the manuscript and useful comments.

\section{References}

[1] S. Eilenberg and D. Montgomery, Fixed point theorems for multi-valued transformations, Amer. J. Math. 68 (1946), 214-222.

[2] S. Eilenberg and N. Steenrod, Foundations of algebraic topology, Princeton Univ. Press, Princeton, New Jersey, 1952.

[3] R. Engelking, General topology, Translated from the Polish by the author, Second edition, Heldermann, Berlin, 1989.

[4] K. Gęba and L. Górniewicz, On the Bourgin-Yang theorem for multivalued maps. I, II, Bull. Polish Acad. Sci. Math. 34 (1986), no. 5-6, 315-328.

[5] L. Górniewicz, Remarks on the Lefschetz fixed point theorem, Bull. Acad. Polon. Sci. Sér. Sci. Math. Astronom. Phys. 21 (1973), 983-989.

[6] _ A Lefschetz-type fixed point theorem, Fund. Math. 88 (1975), no. 2, 103-115.

[7] - Topological fixed point theory of multivalued mappings, Kluwer Acad. Publ., Dordrecht, 1999.

[8] A. Granas and J. Dugundji, Fixed point theory, Springer Monographs in Mathematics, Springer, New York, 2003.

[9] S. Hanai, On closed mappings. II, Proc. Japan Acad. 32 (1956), 388-391.

[10] S. Mardešić, Equivalence of singular and Čech homology for ANR-s. Application to unicoherence, Fund. Math. 46 (1958), 29-45.

[11] M. Nakaoka, Fixed point theorems and its periphery (in Japanese), Iwanami Publishers, 1977.

[12] Note on the Lefschetz fixed point theorem, Osaka J. Math. 6 (1969), 135-142.

[13] Generalizations of Borsuk-Ulam theorem, Osaka J. Math. 7 (1970), 423-441.

[14] Continuous maps of manifolds with involution. I, Osaka J. Math. 11 (1974), $129-145$.

[15] Continuous maps of manifolds with involution. II, Osaka J. Math. 11 (1974), $147-162$.

[16] Equivariant point theorems for involutions, Japan. J. Math. (N.S.) 4 (1978), no. 2, 263-298.

[17] E. H. Spanier, Algebraic topology, Corrected reprint, Springer, New York, 1981.

[18] A. Ju. Volovikov, A generalization of the Borsuk-Ulam theorem, English translation: Math. USSR-Sb. 36 (1980), no. 2, 195-202. 